\title{
An interferometric study of the Fomalhaut inner debris disk
}

\section{Detailed models of the exozodiacal disk and its origin ${ }^{\star}$}

\author{
J. Lebreton ${ }^{1}$, R. van Lieshout ${ }^{2}$, J.-C. Augereau ${ }^{1}$, O. Absil ${ }^{3}$, B. Mennesson ${ }^{4}$, M. Kama ${ }^{2}$, C. Dominik ${ }^{2,5}$, A. Bonsor ${ }^{1}$,

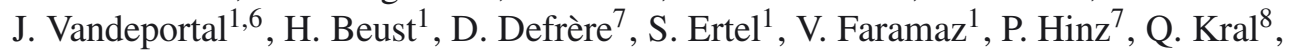 \\ A.-M. Lagrange ${ }^{1}$, W. Liu ${ }^{9}$, and P. Thébault ${ }^{8}$
}

1 UJF-Grenoble 1/CNRS-INSU, Institut de Planétologie et d'Astrophysique de Grenoble (IPAG), UMR 5274, 38041 Grenoble, France e-mail: jeremy.lebreton@obs.ujf-grenoble.fr

2 Astronomical Institute "Anton Pannekoek", University of Amsterdam, PO Box 94249, 1090 GE Amsterdam, The Netherlands

3 Institut d'Astrophysique et de Géophysique, Université de Liège, 17, Allée du 6 Août, B5c B-4000 Sart Tilman, Belgium

4 Jet Propulsion Laboratory, California Institute of Technology, Pasadena, CA 91109, USA

5 Department of Astrophysics/IMAPP, Radboud University Nijmegen, PO Box 9010, 6500 GL Nijmegen, The Netherlands

${ }^{6}$ Département de Physique et Observatoire du Mont-Mégantic, Université de Montréal, CP 6128, Succ. Centre-Ville, Montréal, Québec H3C 3J7, Canada

7 Steward Observatory, University of Arizona, 933 N Cherry Avenue, Tucson, AZ 85721, USA

8 Observatoire de Paris, Section de Meudon, 92195 Meudon Principal Cedex, France

9 Infrared Processing and Analysis Center, California Institute of Technology, Mail Code 100-22, Pasadena, CA 91125, USA

Received 5 March 2013 / Accepted 30 May 2013

\begin{abstract}
Context. Debris disks are thought to be extrasolar analogs to the solar system planetesimal belts. The star Fomalhaut harbors a cold debris belt at $140 \mathrm{AU}$ comparable to the Edgeworth-Kuiper belt, as well as evidence of a warm dust component, unresolved by singledish telescopes, which is suspected of being a bright analog to the solar system's zodiacal dust.

Aims. Interferometric observations obtained with the VLTI/VINCI instrument and the Keck Interferometer Nuller have identified nearand mid-infrared excesses attributed respectively to hot and warm exozodiacal dust residing in the inner few AU of the Fomalhaut environment. We aim to characterize the properties of this double inner dust belt and to unveil its origin.

Methods. We performed parametric modeling of the exozodiacal disk ("exozodi") using the GRaTeR radiative transfer code to reproduce the interferometric data, complemented by mid- to far-infrared photometric measurements from Spitzer and Herschel ${ }^{\star \star}$. A detailed treatment of sublimation temperatures was introduced to explore the hot population at the size-dependent sublimation rim. We then used an analytical approach to successively testing several source mechanisms for the dust and suspected parent bodies. Results. A good fit to the multiwavelength data is found by two distinct dust populations: (1) a population of very small (0.01 to $0.5 \mu \mathrm{m})$, hence unbound, hot dust grains confined in a narrow region $(\sim 0.1-0.3 \mathrm{AU})$ at the sublimation rim of carbonaceous material; (2) a population of bound grains at $\sim 2 \mathrm{AU}$ that is protected from sublimation and has a higher mass despite its fainter flux level. We propose that the hot dust is produced by the release of small carbon grains following the disruption of dust aggregates that originate in the warm component. A mechanism, such as gas braking, is required to further confine the small grains for a long enough time. In situ dust production could hardly be ensured for the age of the star, so we conclude that the observed amount of dust is triggered by intense dynamical activity.

Conclusions. Fomalhaut may be representative of exozodis that are currently being surveyed at near and mid-infrared wavelengths worldwide. We propose a framework for reconciling the "hot exozodi phenomenon" with theoretical constraints: the hot component of Fomalhaut is likely the "tip of the iceberg" since it could originate in the more massive, but fainter, warm dust component residing near the ice line. This inner disk exhibits interesting morphology and can be considered a prime target for future exoplanet research.
\end{abstract}

Key words. techniques: interferometric - zodiacal dust - infrared: planetary systems - stars: individual: Fomalhaut radiative transfer - circumstellar matter

\section{Introduction}

During the past few years, the increasing number of smaller exoplanets and fainter debris disks have revealed that extrasolar

\footnotetext{
* Appendices are available in electronic form at http://www . aanda.org

$\star \star$ Herschel Space Observatory is an ESA space observatory with science instruments provided by European-led Principal Investigator consortia and with important participation from NASA.
}

analogs to our solar system may be common, and yet, little is known about the architecture of the very inner parts of planetary systems. A distinguishable feature of the inner solar system is the existence of the zodiacal cloud composed of small ( 1 to $100 \mu \mathrm{m}$ ) dust grains (Grün 2007; Rowan-Robinson \& May 2013), which are thought to come from the disruption and erosion of comets, asteroids, and Kuiper belt objects (e.g. Nesvorný et al. 2010). Dusty debris disks orbiting other stars than the Sun were first detected by their excess emission in the midor far-infrared (IR), and could then be imaged at visible to 
submillimeter wavelengths. A few warm disks comparable to the zodiacal cloud have been found by space observatories around mature stars via their photometric excess emission at mid-IR wavelengths (Beichman et al. 2005; Lawler et al. 2009; Lisse et al. 2012); but their characterization suffers from insufficient spatial resolution and large photometric uncertainties.

Recent developments in high-angular resolution interferometry have offered powerful tools for characterizing exozodiacal disks (exozodis), which reside in the close environment (typically less than $3 \mathrm{AU}$ ) of a large fraction of nearby stars. Large efforts have indeed been made to detect exozodis with nearand mid-IR interferometers worldwide, notably at the VLTI (Absil et al. 2009), the Keck Interferometer (Millan-Gabet et al. 2011) or the CHARA array at Mount-Wilson (Absil et al. 2006; Defrère et al. 2011b). Ongoing surveys in the near-infrared $(K<$ 5) with the CHARA/FLUOR (Coudé du Foresto et al. 2003) and VLTI/PIONIER (Le Bouquin et al. 2011) interferometers indicate that as much as $\sim 30 \%$ of nearby AFGK mainsequence stars may harbor hot (typically 1000-2000K) circumstellar dust within a few $\mathrm{AU}$ at the $1 \%$ level with respect to photospheric emission (Absil et al., in prep.; Ertel et al., in prep.). Conversely, only $\sim 12 \%$ of the surveyed main sequence stars have been found to harbor mid-infrared excesses (i.e., warm dust) with nulling interferometry (Millan-Gabet et al. 2011).

From a theoretical point of view, the prevalence of these hot excesses around main sequence stars is not understood. Radiative transfer analysis identifies very hot and small refractory grains close to the sublimation limit that should be radiatively blown out over timescales of weeks. Yet they represent typical masses of $10^{-8}$ to $10^{-10} M_{\oplus}$ that need to be delivered by equivalent masses of dust-producing planetesimals (e.g. Defrère et al. 2011a). Being much more massive than the zodiacal cloud, these hot exozodis are difficult to reconcile with the steady-state collisional evolution of parent body belts (Wyatt 2007).

One famous example of a dusty planetary system is the one surrounding the nearby $(7.7 \mathrm{pc})$ A3V star Fomalhaut ( $\alpha$ PsA, HD 216956). This young main sequence star (440 $\pm 40 \mathrm{Myr}$, Mamajek 2012) is well known for its prominent, 140 AU-wide, debris belt that was first resolved in scattered light with HST/ACS revealing a sharp inner edge and side-to-side brightness asymmetry suggestive of gravitational sculpting by a massive planet (Kalas et al. 2005; Quillen 2006). A point source attributed to the suspected planet was later detected in the optical at the predicted location, moving along its orbit over multiple epochs (Kalas et al. 2008). The absence of detection in the nearand mid- (thermal) infrared range (Janson et al. 2012) resulted in a controversial status for Fomalhaut b. More recent studies confirm the detections of a companion at $118 \mathrm{AU}$ and interpret the various constraints as a large circumplanetary dust disk orbiting a hidden subjovian planet, but do not exclude an isolated dust cloud originating from a recent collision between planetesimals (Currie et al. 2012; Galicher et al. 2013) ${ }^{1}$. The cold planetesimal belt is indeed collisionally very active as shown by the recent analysis of resolved images and photometry at far-infrared to submillimeter wavelengths from ALMA (Boley et al. 2012) and Herschel/PACS and SPIRE (Acke et al. 2012). Using radiative transfer modeling, Acke et al. (2012) argue that a dust production rate of $\sim 3 \times 10^{-5} M_{\oplus} /$ year is needed to justify the measured amount of blow-out grains, and estimate that the region interior to the cold belt at $140 \mathrm{AU}$ are not devoid of material.

\footnotetext{
Kalas et al. (2013) recently announced a fourth epoch detection of Fomalhaut $b$. They argue in favor of an object larger than a dwarf planet evolving on a very eccentric orbit (see Sect. 6).
}

The Herschel/PACS $70 \mu \mathrm{m}$, as well as the ALMA image, identify an unresolved excess in the vicinity of the star that was previously reported in the mid-infrared by Stapelfeldt et al. (2004) based on Spitzer/MIPS imaging and IRS spectroscopy. $\mathrm{Su}$ et al. (2013) recently analyzed the IRS and PACS data and concluded on the presence of a warm debris belt with a blackbody temperature of $\sim 170 \mathrm{~K}$. However, these facilities lack the spatial resolution and accuracy needed to characterize this warm component.

Near- and mid-infrared long baseline interferometers offer the appropriate tools to study the Fomalhaut exozodi with enough contrast and resolution, free of any modeling assumptions on the stellar spectrum.

The present paper carries out a thorough analysis of the Fomalhaut inner debris disk. It is the last one of a series initiated by Absil et al. (2009, henceforth Paper I) - who presented the VLTI/VINCI detection of hot excess in the close environment of the star - followed by a mid-infrared characterization using the Keck Interferometer Nuller (KIN) by Mennesson et al. (2013, henceforth Paper II). In Paper I, we presented the clear $K$-band $(2.18 \mu \mathrm{m})$ detection of a short-baseline visibility deficit. It is best explained by circumstellar emission emanating from within 6 AU of the star with a relative flux level of $0.88 \pm 0.12 \%$. In Paper II, we presented multiwavelength measurements performed across the $N$-band ( 8 to $13 \mu \mathrm{m}$ ) using the technique of nulling interferometry. A small excess is resolved within $\sim 2 \mathrm{AU}$ from the star, with a mean null depth value of $0.35 \% \pm 0.10 \%{ }^{2}$. Preliminary modeling shows that the near- to mid-infrared excesses can only be explained by two distinct populations of dust emitting thermally; small $(\sim 20 \mathrm{~nm})$ refractory grains residing at the sublimation distance of carbon are responsible for the near-infrared emission, while $>1 \mu \mathrm{m}$ grains located further than the silicate sublimation limit produce most of the mid-infrared excesses.

The paper is organized as follows. In Sects. 2 and 3, we present our radiative transfer model of optically thin disks, and introduce a new prescription for calculating the sublimation distance of dust grains in an exozodi. Results of the modeling of the inner Fomalhaut debris disk, based on multiwavelengths observations, are detailed in Sect. 4. The mechanisms that produce and preserve the hot grains, as well as the connection with the warm and the cold belt are discussed in Sect. 5. We discuss further our results and attempt to place the Fomalhaut exozodi in the context of its planetary system in Sect. 6. We finally summarize our main findings in Sect. 7.

\section{A schematic exozodiacal disk model}

In this section, we elaborate a schematic model of an optically thin exozodiacal dust disk. The model is implemented in the GRaTeR code originally developped by Augereau et al. (1999). It makes hardly any a priori assumptions regarding the nature of the grains and their production. Because of the limited constraints on detected exozodiacal disks, we restrain the model to a 2D geometry. The disk surface density and grain size distribution are parametrized with simple laws to limit the number of free parameters. This allows us to explore a broad range of disk properties using a Bayesian inference method.

\footnotetext{
2 We note that the true astrophysical excess could be larger because some dust emission may be removed by the destructive fringes of the interferometer. The nuller transmission pattern is accounted for in the models discussed afterwards.
} 


\subsection{Scattered light and thermal emission}

We consider a population of dust grains at a distance $r$ from the star and with a differential size distribution $\mathrm{d} n(r, a)$, where $a$ is the grain radius. In a self-consistent description of a collisional evolution of debris disks, the spatial and size distribution cannot be formally separated (Augereau et al. 2001; Krivov et al. 2006; Thébault \& Augereau 2007). Since we know little about the properties and origin of observed exozodiacal disks, we assume here for simplicity that the dependence of the size distribution on the distance $r$ essentially reflects the size-dependent sublimation distance of the grains. Since exozodiacal grains may reach very high temperatures, sublimation can prevent the smallest grains from surviving in regions where larger ones can remain, thereby truncating the size distribution at its lower end. We therefore write the differential size distribution $\mathrm{d} n(r, a)$ as follows:

$$
\begin{aligned}
& \mathrm{d} n(r, a)=H\left(a-a_{\mathrm{sub}}(r)\right) \mathrm{d} n(a) \\
& \text { with } \int_{a_{\min }}^{a_{\max }} \mathrm{d} n(a)=1
\end{aligned}
$$

where $a_{\min }$ and $a_{\max }$ are the minimum and maximum grain sizes respectively, $H\left(a-a_{\text {sub }}(r)\right)$ is the Heaviside function (assuming $H(0)=1)$ and $a_{\mathrm{sub}}(r)$ is the sublimation size limit at distance $r$ from the star. Details of the calculation for sublimation will be given in Sect. 3 .

At wavelength $\lambda$, the dust population thermally emits a flux

$\Phi_{\text {th }}(\lambda, r)=\int_{a_{\text {min }}}^{a_{\max }} B_{\lambda}\left(T_{\mathrm{d}}(a, r)\right) \frac{\sigma_{\mathrm{abs}}(\lambda, r, a)}{4 d_{\star}^{2}} \mathrm{~d} n(r, a)$

where $d_{\star}$ is the distance of the observer to the star, $T_{\mathrm{d}}(a, r)$ is the grain temperature and $B_{\lambda}$ is the Planck function. In the above equation, we implicitly assumed that grains thermally emit isotropically. The absorption cross-section $\sigma_{\mathrm{abs}}(\lambda, r, a)$ reads

$\sigma_{\mathrm{abs}}(\lambda, r, a)=4 \pi a^{2} Q_{\mathrm{abs}}\left(\frac{2 \pi a}{\lambda}, \lambda, r\right)$

where $Q_{\mathrm{abs}}$ is dimensionless absorption/emission coefficient that depends on the size parameter $2 \pi a / \lambda$, on $\lambda$ through the wavelength-dependent optical constants, and on the distance to the star as the grain composition may depend on $r$. The grain temperature $T_{\mathrm{d}}(a, r)$ is obtained by solving in two steps the thermal equilibrium equation of a grain with the star. For any grain size $a$, we first calculate the equilibrium distance $r$ for a broad range of grain temperatures $T_{\mathrm{d}}$ knowing the $Q_{\mathrm{abs}}$ value

$r\left(a, T_{\mathrm{d}}\right)=\frac{d_{\star}}{2} \sqrt{\frac{\int_{\lambda} Q_{\mathrm{abs}} F_{\star}(\lambda) \mathrm{d} \lambda}{\int_{\lambda} Q_{\mathrm{abs}} \pi B_{\lambda}\left(T_{\mathrm{d}}\right) \mathrm{d} \lambda}}$

where $F_{\star}(\lambda)$ is the stellar flux at Earth. The $r\left(a, T_{\mathrm{d}}\right)$ function is then numerically reversed to get $T_{\mathrm{d}}(a, r)$.

Assuming isotropic scattering for simplicity, a dust population at distance $r$ from the star scatters a fraction of the stellar flux at wavelength $\lambda$ is given by

$\Phi_{\mathrm{sc}}(\lambda, r)=F_{\star}(\lambda) \frac{\sigma_{\mathrm{sca}}(\lambda, r)}{4 \pi r^{2}}$

with $\sigma_{\text {sca }}$ the mean scattering cross section

$\sigma_{\text {sca }}(\lambda, r)=\int_{a_{\min }}^{a_{\max }} \pi a^{2} Q_{\text {sca }}\left(\frac{2 \pi a}{\lambda}, \lambda, r\right) \mathrm{d} n(r, a)$

and $Q_{\text {sca }}$ the dimensionless scattering coefficient.
The total flux emitted at wavelength $\lambda$ by the dust population finally reads

$\Phi(\lambda, r)=\Phi_{\mathrm{sc}}(\lambda, r)+\Phi_{\mathrm{th}}(\lambda, r)$.

\subsection{Synthetic observations}

We synthesize single aperture photometric observations of non edge-on exozodiacal dust disks at wavelength $\lambda$ by calculating the integral

$$
\begin{aligned}
& \Phi(\lambda)=\int_{r=0}^{\infty} \Phi(\lambda, r) \Sigma(r) \times 2 \pi\langle\mathrm{FOV}\rangle_{\theta}(r) r \mathrm{~d} r \\
& \text { with }\langle\mathrm{FOV}\rangle_{\theta}(r)=\int_{\theta=0}^{2 \pi} \operatorname{FOV}(\rho(r, \theta)) \frac{\mathrm{d} \theta}{2 \pi} \\
& \text { and } \rho(r, \theta)=r \sqrt{1-\cos ^{2} \theta \sin ^{2} i}
\end{aligned}
$$

where $r$ and $\theta$ are cylindrical coordinates in the disk plane and $\rho(r, \theta)$ the projected distance to the star in the sky plane, $\Sigma(r)$ is the dust surface number density of the exozodiacal disk, assumed to be axisymmetrical and $i$ is the disk inclination with respect to the sky plane ( $i=0$ for pole-on geometry). In the above equations, we implicitly assumed that the instrument beam profile FOV only depends upon $\rho$, the projected distance to the star in the sky plane. The $\langle\mathrm{FOV}\rangle_{\theta}(r)$ function gives the azimuthally averaged telescope transmission along circles of radius $r$ in the exozodiacal disk frame.

Most single aperture telescopes such as Spitzer or Herschel, have sufficiently large beams (or slits in case of spectroscopy), which intercept the entire exozodiacal dust emission. In such a case, the exozodiacal flux emission can be obtained by taking $\langle\mathrm{FOV}\rangle_{\theta}(r)=1$ in Eq. (8). On the other hand, coherent near- and mid-IR interferometric observations, such as those obtained with the VLTI/VINCI and CHARA/FLUOR instruments, have much smaller fields of view (FOV) and the actual transmission profiles of the interferometric instruments on the sky plane is important ${ }^{3}$.

\subsection{Grain properties}

In the solar system, the zodiacal dust particles are thought to originate in tails and disruption of comets, or to be produced when asteroids collide. Both interplanetary and cometary dust particles are composed of silicates and carbonaceous material, and zodiacal cloud dust particles are expected to be made of similar material. In this model, we consider mixtures of silicates and carbonaceous material, and calculate their optical properties with the Mie theory valid for hard spheres. The optical index of the mixture is calculated using the Bruggeman mixing rule, given a relative volume fraction $v_{\mathrm{C}} / v_{\mathrm{Si}}$ of carbonaceous grains. In the disk regions where the grain temperature is between the sublimation temperature of silicates and carbon, the volume of silicates is replaced by vacuum, mimicking porous carbonaceous grains. The optical constants and grain bulk densities used in this study are summarized in Table 1.

We adopt a power-law differential size distribution

$\mathrm{d} n(a)=\left(\frac{1-\kappa}{a_{\max }^{1-\kappa}-a_{\min }^{1-\kappa}}\right) a^{-\kappa} \mathrm{d} a$

for grain radii $a$ between $a_{\min }$ and $a_{\max }$. The maximum grain size has no impact at the wavelengths considered and cannot be

\footnotetext{
3 See Fig. 1 of Paper II for the KIN transmission map.
} 
Table 1. Material properties.

\begin{tabular}{lcc}
\hline \hline & Carbonaceous material & Silicates \\
Nickname & $\mathrm{C}$ & $\mathrm{Si}$ \\
\hline \multicolumn{2}{l}{ Thermodynamical properties } \\
Material type & Graphite & Olivine \\
\hline$A[\mathrm{cgs}]$ & 37215 & 28030 \\
$B[\mathrm{cgs}]$ & 1.3028 & 12.471 \\
$\mu\left[\mathrm{m}_{\mathrm{p}}\right]$ & 12.0107 & 172.2331 \\
$\rho\left[\mathrm{g} . \mathrm{cm}^{-3}\right]$ & 1.95 & 3.5 \\
\hline Optical properties & & \\
Material type & aC ACAR & Astrosilicates \\
Reference & Zubko et al. (1996) & Draine (2003) \\
\hline
\end{tabular}

Notes. The thermodynamical constants A and B are tabulated values from Kama et al. (2009) and Zavitsanos \& Carlson (1973).

constrained with the adopted modeling approach. It is thus fixed to $a_{\max }=1 \mathrm{~mm}$ in the rest of this study.

\subsection{Fitting strategy}

The adopted fitting stategy is based on a Bayesian inference method described in Lebreton et al. (2012). For that purpose, a grid of models is created. For each set of parameters, the goodness of the fit is evalutated with a reduced $\chi_{\mathrm{r}}^{2}$ that is transformed into probabilities assuming a Gaussian likelihood function $\left(\propto \mathrm{e}^{-\chi_{\mathrm{r}}^{2} / 2}\right)$ for Bayesian analysis. Marginalized probability distributions for each free parameter are then obtained by projection of these probabilities onto each dimension of the parameter space.

In order to limit the number of free parameters, we adopt a two power-law radial profile for the surface density

$\Sigma(r)=\Sigma_{0} \sqrt{2}\left[\left(\frac{r}{r_{0}}\right)^{-2 \alpha_{\mathrm{in}}}+\left(\frac{r}{r_{0}}\right)^{-2 \alpha_{\mathrm{out}}}\right]^{-1 / 2}$.

This corresponds to a smooth profile, with an inner slope $r^{\alpha_{\text {in }}}$ $\left(\alpha_{\text {in }}>0\right)$ that peaks at about $r_{0}$, and that falls off as $r^{\alpha_{\text {out }}}$ $\left(\alpha_{\text {out }}<0\right)$ further to $r_{0}$.

The parameter space explored here is listed in Table 4 of Paper II and recalled in Sect. 4.2. Each set of parameters defines an emission spectrum, 2D emission, and scattered light maps that are flux-scaled by searching the optimal $\Sigma_{0}$ value (surface density at $r=r_{0}$ ) that gives the best fit to the panchromatic observations. The range of $a_{\text {min }}$ values includes sizes that are far below the blow-out size limit for grains about Fomalhaut.

\section{Dust sublimation model}

\subsection{Sublimation temperatures}

We implement a new method of calculating the sublimation temperature $T_{\text {sub }}$ of a dust grain as a function of its size and not only of its composition, based on the method introduced by Kama et al. (2009) to study the inner rim of protoplanetary disks. We first model a grain as an homogeneous sphere of radius $a$ composed of a single material of density $\rho_{\mathrm{d}}$. To ensure the stability of a grain (i.e. no net change of its size), the flux of particles (carbon/silicates monomeres) escaping from its surface must equal the flux of particles coming in from the ambient medium. From the kinetic theory of gases, the number of particles accreted onto and evaporated from the grain surface per unit time and unit surface reads respectively (Lamy 1974)

$$
\begin{aligned}
& F_{\text {accre }}=\frac{P_{\text {gas }}}{\sqrt{2 \pi \mu m_{\mathrm{u}} k_{\mathrm{B}} T_{\text {gas }}}} \\
& F_{\text {evap }}=-\frac{P_{\text {eq }}}{\sqrt{2 \pi \mu m_{\mathrm{u}} k_{\mathrm{B}} T_{\text {gas }}}}
\end{aligned}
$$

where $P_{\text {eq }}$ is the gas saturation partial pressure, $P_{\text {gas }}$ the partial pressure of the ambient gas medium, $T_{\text {gas }}$ the gas temperature assumed equal to the dust temperature $T_{\mathrm{d}}$ here, $\mu m_{\mathrm{u}}$ the mean molecular weight and $k_{\mathrm{B}}$ the Boltzmann constant. Introducing an efficiency factor $\alpha$ (constrained by laboratory experiments), the mass of a grains of radius $a$ then evolves as

$\frac{\mathrm{d} m}{\mathrm{~d} t}=\alpha\left(F_{\text {accre }}-F_{\text {evap }}\right) \times \mu m_{\mathrm{u}} 4 \pi a^{2}$

Injecting the ideal gas law $P=\rho k_{\mathrm{B}} T / \mu m_{\mathrm{u}}$ yields:

$\frac{\mathrm{d} m}{\mathrm{~d} t}=\alpha a^{2} \sqrt{\frac{8 \pi k_{\mathrm{B}} T_{\mathrm{d}}}{\mu m_{\mathrm{u}}}}\left(\rho_{\mathrm{gas}}-\rho_{\mathrm{eq}}\right)$

$\frac{\mathrm{d} a}{\mathrm{~d} t}=\frac{\alpha}{\rho_{\mathrm{d}}} \sqrt{\frac{k_{\mathrm{B}} T_{\mathrm{d}}}{2 \pi \mu m_{\mathrm{u}}}}\left(\rho_{\mathrm{gas}}-\rho_{\mathrm{eq}}\right)$.

For the purpose of this study, the grains are assumed to lie in empty space $\left(\rho_{\text {gas }}=0\right)$ and their temperature equals by definition the sublimation temperature $T_{\text {sub }}$. The gas density at saturation pressure $\rho_{\text {eq }}$ is given by the Clausius-Clapeyron equation

$\log _{10} \rho_{\mathrm{eq}}=B-\frac{A}{T_{\text {sub }}}-\log _{10} T_{\text {sub }}^{-\mathrm{C}}$

where the thermodynamical quantities $A$ and $B$ are determined from laboratory measurements and $C=-1$, as discussed by Kama et al. (2009). Integrating equation 17 from the initial grain size $a$ to 0 , under the assumption that the grain temperature $T_{\mathrm{d}}=T_{\text {sub }}$ does not vary significantly during the sublimation process, yields

$\frac{a}{t_{\text {sub }}}=\frac{\alpha}{\rho_{\mathrm{d}}} \sqrt{\frac{k_{\mathrm{B}} T_{\text {sub }}}{2 \pi \mu m_{\mathrm{u}}}} 10^{\mathrm{B}-\frac{\mathrm{A}}{\mathrm{T}_{\text {sub }}}-\log _{10} T_{\text {sub }}}$

where we define $t_{\text {sub }}$ as the time needed to sublimate an entire grain.

This equation relates the sublimation temperature of a grain to its size and to a sublimation timescale. Although determining this timescale without a time-dependent approach is tricky, we will see in Sect. 3.2 that our parametric approach allows to tackle the issue based on simple assumptions. Beforehand we need to extend the sublimation model to the case of multi-material grains.

We recall that our objective is to interpret real observations of individual debris disks. Fitting the spectral and spatial observables of these disks requires to solve accurately a radiative transfer problem and to model the optical properties of the materials at stake. To achieve this, the GRaTeR code handles grains made of multiple (in particular carbonaceous and silicate) materials by considering homogeneous spheres with optical constants obtained by means of an effective medium theory. Porous grains are represented by a compact sphere in which one of the "materials" is vacuum. When one of the material reaches its sublimation temperature, it is automatically replaced by vacuum. This is in 


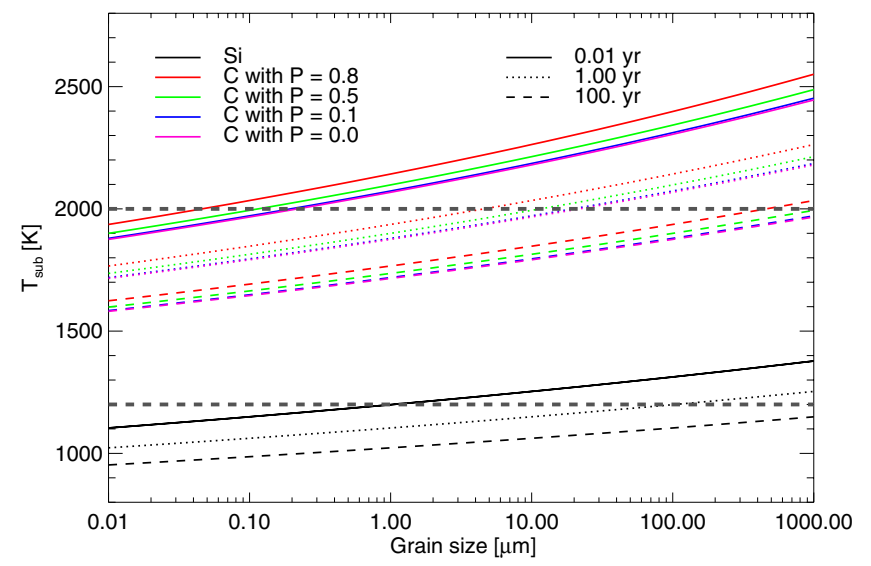

Fig. 1. Sublimation temperatures of carbon and silicate as a function of grain size with several possible survival timescales, and assuming different porosities for carbonaceous grains or equivalently different volume fractions of silicate. The horizontal dashed lines show the constant $T_{\text {sub }}$ values often used in past studies.

particular the case for the grains that lie very close to the star: then a silicate-carbon mixture becomes a porous carbon grain. This approach proved efficient at reproducing the optical behavior of astronomical dust grains in various situations.

We consider homogeneously distributed mixtures of silicate and carbon characterized by a volume fraction $v_{\mathrm{C}} / v_{\mathrm{Si}}$. We anticipate that silicate will sublimate at lower temperatures than carbon whatever the grain size: silicates will have vanished entirely before the carbon starts its sublimation. As a first step the sublimation occurs as if the grains were entirely made of silicates. In a second step, the grains take the form of a carbon matrix filled with cavities. For spheroids, the porosity can be defined as the filling factor of vacuum $\mathcal{P}=\frac{V_{\text {vacuum }}}{V_{\text {grain }}}$. We introduce the porosity by correcting the grain density: $\rho_{\mathrm{d}}(\mathcal{P}) \rightarrow(1-\mathcal{P}) \rho_{\mathrm{d}}$ in Eq. (19) which is then solved using the density of the porous carbonaceous leftover.

Equation (19) is solved using the material properties summarized in Table 1, and the solution is inversed numerically to determine $T_{\text {sub }}$ as a function of grain size for different timescales. The sublimation curves for pure silicate and carbon-rich grains $(\mathcal{P} \simeq 0)$ is displayed in Fig. 1, together with that of porous carbonaceous grains mimicking mixtures of $\mathrm{Si}$ and $\mathrm{C}$ for which $\mathrm{Si}$ has sublimated. We observe that porosity tends to increase $T_{\text {sub }}$, as do smaller sublimation timescales. These are interpreted respectively as a loss of efficiency in the sublimation process for lower grain densities, and as a consequence of the exposure time. Compared with the usual constant $T_{\text {sub }}$ approximation, large and small grains have their sublimation temperatures re-evaluated by as much as plus or minus $\sim 20 \%$.

\subsection{Timescales}

From what precedes, we are able to define a size- and composition-dependent estimate for the sublimation temperature of a dust grain. Figure 1 reveals an important deviation from the constant sublimation temperature. However, we first need to know on which timescale the sublimation has to be considered. One can notice that an order of magnitude error on the timescale estimate will result only in a modest shift in the sublimation curve. Sublimation can be expected to alter the steadystate grain size distribution because it introduces an additional (size-dependent) destruction mechanism; nonetheless we take advantage of the fact that we are using a parametric model and

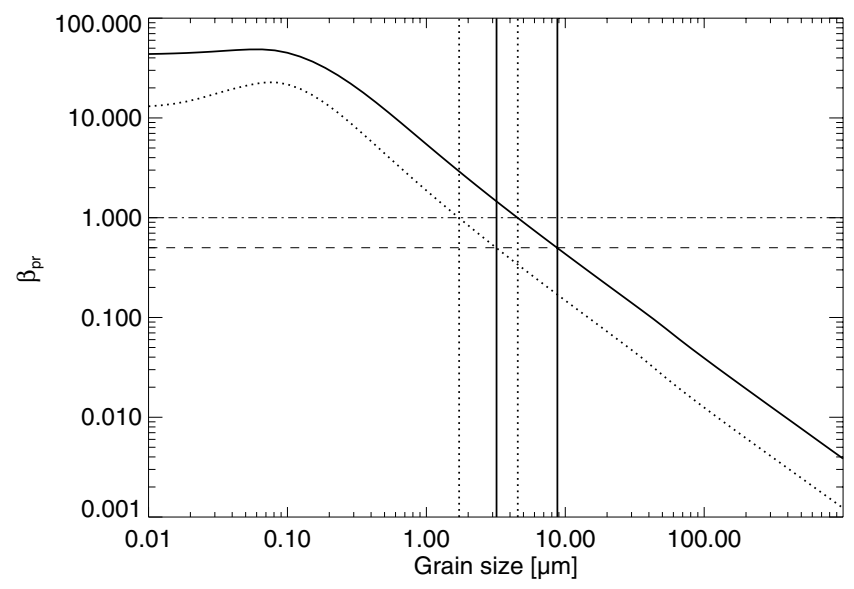

Fig. 2. $\beta$ ratio of a dust grain as a function of its size in the Fomalhaut environment. A 50-50 carbon-silicate mixture is assumed and depicted by the lower curve while the upper curve considers half porous carbon grains. The vertical and horizontal lines mark the $\beta=0.5$ (blowout size for grains released by parent-bodies on initially circular orbits) and $\beta=1.0$ (blowout size for any initial orbit) limits, before and after the sublimation of silicates.

we address only the question: how big must a grain be to survive a temperature $T_{\text {sub }}$ for some time $t$.

A grain lifetime is limited by its removal processes. In a debris disk, the main removal process for bound grains is generally destructive collisions, but when the optical depths and/or the dust stellocentric distance is sufficiently small, PoyntingRobertson (PR) drag can become the dominant effect (Wyatt 2005). Unbound grains are placed on hyperbolic orbits and ejected from the system before they are destroyed: their survival timescale can be equaled to a "blowout timescale". Assuming near-circular orbits for the parent-bodies, the limit between bound and unbound grains is set by $a_{\text {blow }}=a(\beta=1 / 2)$ (Fig. 2 ), where $\beta$ is the size-dependent ratio of radiation pressure to gravitational forces.

Here we want to determine the survival timescale of a grain, in the sublimation zone so depending on its size $a$. The sublimation zone is defined, for each of the materials that the grains are made of, as the interval $\left[d_{1}, d_{2}\right]$ between the minimum and maximum sublimation distances (all grain sizes considered) assuming constant sublimation temperature. $d_{1}$ is essentially independent of the maximum grain size $a_{\max }$ because the sublimation distance does not vary with size for grains larger than a few $\mu \mathrm{m}$ (Fig. 4).

We calculate a preliminary grid of models for each grain composition (with no fine computation of the sublimation temperature), and we identify the grain properties and disk surface density that best fit the observations. This provides an estimate of the vertical optical depths required to calculate the collisional timescale: the mean timescale a barely bound grain $(\beta \simeq 1 / 2)$ can survive in a collision-dominated disk:

$t_{\text {col }}^{0}\left(a_{\text {blow }}\right)=\frac{t_{\text {orbit }}}{2 \pi \tau_{\perp}^{\text {geo }}}$

with $t_{\text {orbit }}$ the orbital period and $\tau_{\perp}^{\text {geo }}$ the vertical geometrical optical depth at distance $d_{1}$, a distance representative of the sublimation distance of bound grains.

For larger grains, the collision timescale is scaled with size as (Thébault \& Augereau 2007)

$t_{\mathrm{col}}(a)=t_{\mathrm{col}}^{0}\left(\frac{a}{a_{\mathrm{blow}}}\right)^{0.3}$ 


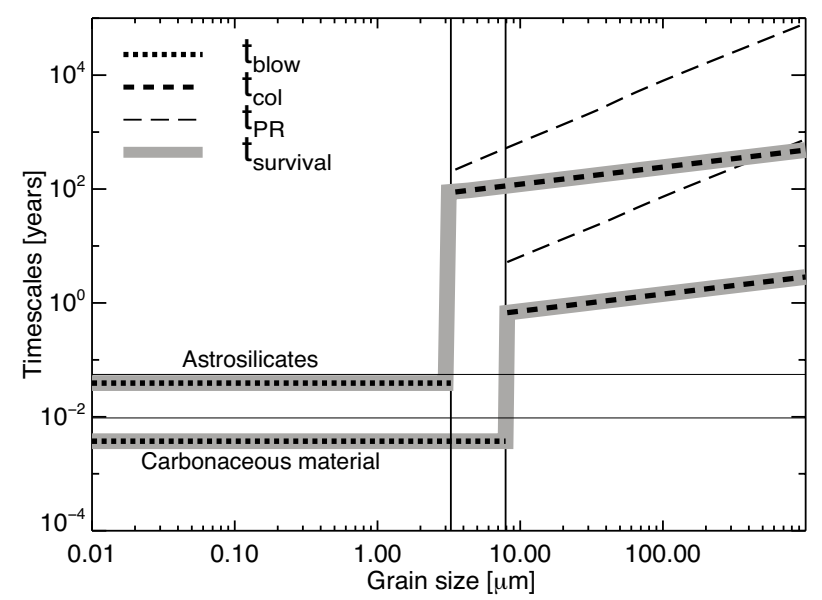

Fig. 3. Examples of survival timescales against collisions, PR drag and radiation pressure blowout calculated for an exemplary grain composition $\left(v_{\mathrm{C}}=v_{\mathrm{Si}}\right.$ for silicate, $\mathcal{P}=50 \%$ for carbonaceous material). For each effect, the upper curve corresponds to silicate and the lower curve to carbon, each in its sublimation zone and for the disk properties derived from preliminary modeling. The horizontal black lines give the orbital periods for reference. The vertical lines mark the blowout size before (left) and after (right) silicate sublimates.

where $t_{\mathrm{col}}$ depends on the sublimation zone of a given material and on the assumed surface density profile.

We define the PR drag timescale as the time needed for bound grains to spiral from the outer edge of the sublimation zone, to the inner edge of the sublimation zone (Wyatt 2005)

$t_{\mathrm{PR}}(a)=400 \beta(a) \frac{\left(d_{2}-d_{1}\right)^{2}}{M_{*}}$.

As soon as they are produced, unbound grains $(\beta>1 / 2)$ are placed onto hyperbolic orbits, they are ejected from the sublimation zone and eventually from the field of view. In the sublimation zone, the hyperbolic orbit is approximated by rectilinear uniform motion; we assume the grains are produced at the inner edge of the sublimation zone $d_{1}$ with initial velocity $v_{\mathrm{Kep}}\left(d_{1}\right)$. A grain will travel typically a distance between $\sqrt{d_{2}^{2}-d_{1}^{2}}(\beta(a)=1)$ and $d_{2}-d_{1}(\beta \gg 1 / 2)$. An estimate of the blowout timescale is given by the average between these two extremes (see Eq. (31) and Appendix A for a more general estimate)

$t_{\text {blow }}=\frac{1}{2}\left[\frac{\left(d_{2}^{2}-d_{1}^{2}\right)^{0.5}}{v_{\text {Kep }}}+\frac{d_{2}-d_{1}}{v_{\text {Kep }}}\right]$.

Eventually, the sublimation timescale is equaled to the survival timescale, namely the longest time a grain can be exposed to sublimation, according to

$t_{\mathrm{sub}}(a)=\left\{\begin{array}{cl}\min \left(t_{\mathrm{col}}(a), t_{\mathrm{PR}}(a)\right), & \text { for } a>a_{\mathrm{blow}} \\ t_{\mathrm{blow}}, & \text { for } a \leq a_{\mathrm{blow}}\end{array}\right.$

A representative example for Fomalhaut is shown in Fig. 3. The properties of the silicate population and of the carbon population described in details in Sect. 4 have been assumed. $t_{\text {sub }}(C)$ is always smaller than $t_{\text {sub }}(\mathrm{Si})$ because of the respective locations of the two grain populations. The sharp discontinuity between the survival timescale of bound and unbound grains translates into a big jump in the sublimation temperatures and then the distances at the blowout limit. We stress that Fig. 3 only illustrates the survival time in the sublimation zone, it should not be used to find the dominant mechanism in the entire dust disk.
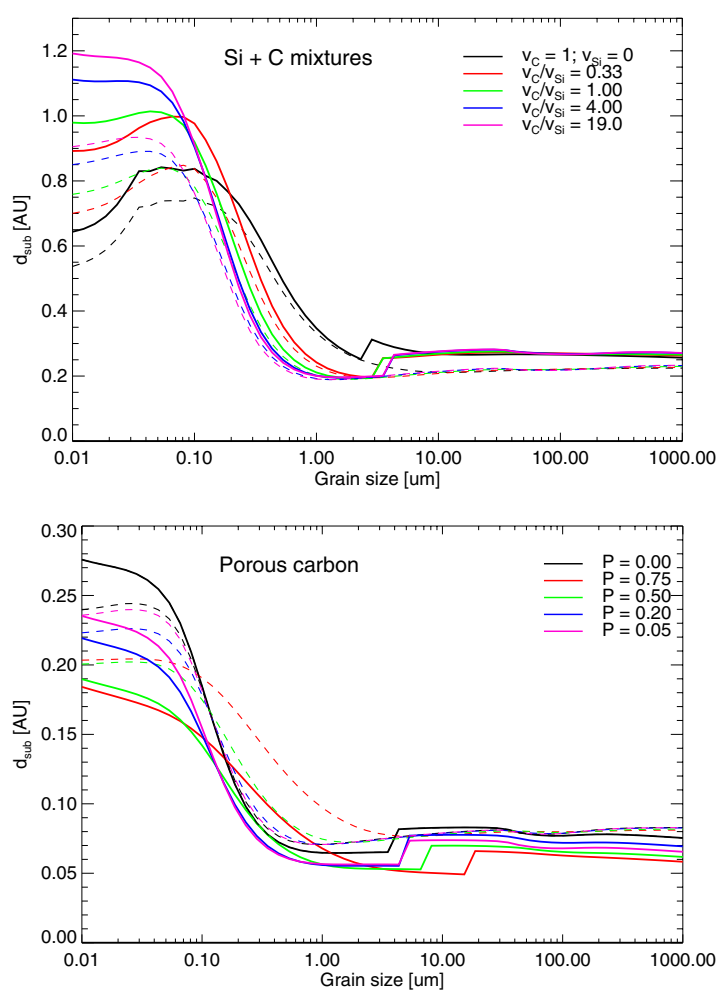

Fig. 4. Sublimation distances of silicate (top panel) and carbonaceous material (bottom panel) in composite grains $(\mathrm{C}+\mathrm{Si}$ or $\mathrm{C}+$ vacuum respectively) as a function of grain size in the Fomalhaut environment, with either a constant (dashed line) or a size-dependent (solid line) sublimation temperature. Relevant disk properties obtained from preliminary modeling are used (Sect. 4). Size-dependent sublimation temperatures are a function of the size-dependent survival timescales shown in Fig. 3 causing jumps at the material-dependent blowout limit.

\subsection{Sublimation distances}

The GRaTeR code handles the optical properties of various materials. Broad-band spectra and excesses attributed to warm circumstellar dust disks are well matched using materials of the silicate family or carbonaceous materials. For each of them, we use respectively the thermodynamical quantities associated to amorphous olivine $\left(\mathrm{MgFeSiO}_{4}\right)$ and graphite $(\mathrm{C})$. They are indicated in Table 1.

After equaling the sublimation timescale to a timescale relevant for each grain (Sect. 3.2), we calculate the thermal equilibrium using the usual formula (Eq. (4)). This provides the size dependent-sublimation distance presented in Fig. 4 for a few representative material mixtures. The overall shape of the curves is dictated by the thermal equilibrium distances. The net effect of the new model is generally an alteration of the sublimation distances of the small $(<10 \mu \mathrm{m})$ grains, depending on the timescales used for each model. The inner edge of the sublimation is close to $0.2 \mathrm{AU}$ for silicate, $0.05 \mathrm{AU}$ for carbon, while their outer edge lies respectively at $0.7-1.2 \mathrm{AU}, 0.18-0.25 \mathrm{AU}$ respectively.

\subsection{Sublimation model: summary}

We presented an innovative model that is used to calculate size-dependent sublimation distances of dust grains depending on their compositions, porosities and survival timescales. The method can be summarized as follows

1. A preliminary grid of models is adjusted to the data assuming fixed sublimation temperatures and solving the 
size-dependent equilibrium temperatures. This provides estimates of the dust disk location and density (Paper II).

2. Size-dependent survival timescales are estimated by searching for the most efficient effect between destructive collisions, PR drag and photo-gravitational blowout (Fig. 3).

3. Size-dependent sublimation temperatures are calculated from the kinetic theory of gases and thermodynamics as a function of the timescale for when a grain is exposed to sublimation (Fig. 1).

4. This sublimation timescale is equaled to the survival timescale for each grain yielding a size-dependent sublimation temperature that accounts for each grain specific dynamical regime. In particular, this causes a discontinuity at the blowout limit (Fig. 2).

5. Finally, size-dependent sublimation distances are reevaluated by solving the equilibrium distance of each grain knowing its specific sublimation temperature (Eq. (4)).

\section{Application and results}

\subsection{Observational constraints and star properties}

The model presented above is now confronted with the observations of Fomalhaut already presented in Table 3 of Paper II. Preliminary analysis of the near-infrared VLTI/VINCI excess, of mid-infrared KIN null excesses, complemented by (low accuracy) spectrophotometric data, suggested the coexistence of two populations of dust in the Fomalhaut environment located within the field of view of the interferometers ( $\sim \mathrm{AU}$ and $\sim 2$ AU HWHM for FLUOR and KIN respectively). This architecture is imposed by the high level of the K-band excess with respect to $N$-band, and the inversion of the null excess slopes upon $\sim 10 \mu \mathrm{m}$, with a rising profile toward the longer wavelengths. Stark et al. (2009) observed a similar break around $10 \mu \mathrm{m}$ in the Keck null depths of 51 Ophiuchus. They were only able to reproduce it with a double-population of dust grains, however with much larger spatial scales due to the distance of the star. In Paper II, the two dust populations of Fomalhaut were adjusted separately and the model suffered from an inaccurate prescription for the sublimation distances. In the present study we propose a self-consistent modeling of the exozodi and we characterize precisely the properties of the emitting grains and their location. We assume the disk position angle and inclination to equal those of the cold ring $\left(i=65.6^{\circ}, \mathrm{PA}=156^{\circ}\right)$. A NextGen photosphere (Hauschildt et al. 1999) is scaled to the $V$ magnitude of Fomalhaut $\left(m_{\mathrm{V}}=1.2 \mathrm{mag}\right)$ in order to model the total flux received by the grains. It also serves to estimate the excesses attributable to the disk in the photometric data. The interferometric observables on the other hand are independent from the chosen star spectrum.

\subsection{Data and modeling strategy}

We adopt a strategy in which we fit (1) the "hot dust ring" ( $\lesssim 0.4 \mathrm{AU})$ probed by the shortest wavelengths data, using the same data subset as Paper II (most importantly the VINCI $2.18 \mu \mathrm{m}$ excess), and then (2) the "warm dust belt" probed by the KIN nulls from 8 to $13 \mu \mathrm{m}$ and mid- to far-infrared photometric measurements of the warm "on-star" excess (Figs. 5 and 6).

These photometric measurements are derived from unresolved observations of the inner Fomalhaut debris disk, well differentiated from the contribution of the cold belt by

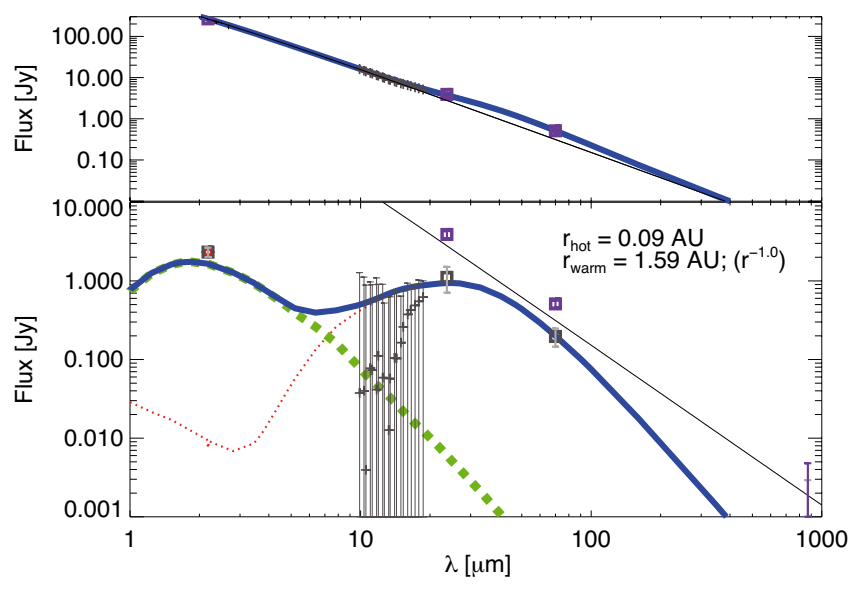

Fig. 5. Measured spectral energy distribution, and SED of the bestfitting double-dust belt model (green: hot ring, red: warm belt, blue: total). Top panel: global SED including a NextGen photosphere model (solid black line), bottom panel: circumstellar excess emission. From left to right, thick squares denote the VLTI/VINCI $2.18 \mu \mathrm{m}$ excess, MIPS $24 \mu \mathrm{m}$ and Herschel/PACS $70 \mu \mathrm{m}$ photometry with $1 \sigma$ error bars. Spitzer/IRS spectrum (grey crosses, not fitted) and ALMA $870 \mu \mathrm{m}$ photometry (purple cross) are shown as $3 \sigma$ upper limits.
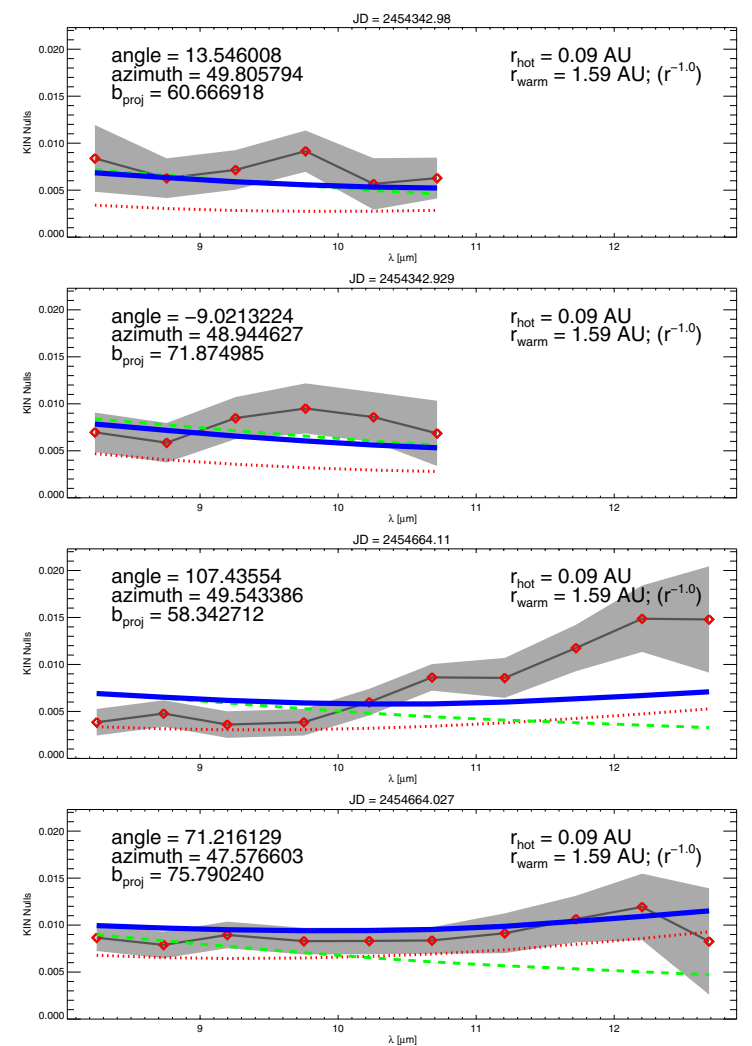

Fig. 6. Measured KIN excess null depths and nulls of the best-fitting double-dust belt model (green: hot ring, red: warm belt, blue: total) for the four data subsets. The grey regions denote the $1 \sigma$ confidence intervals on the data.

Spitzer/MIPS at $23.68 \mu \mathrm{m}\left(F_{24}=3.90 \pm 0.40 \mathrm{Jy}\right.$, Stapelfeldt et al. 2004), Herschel/PACS at $70 \mu \mathrm{m}\left(F_{70}=0.51 \pm 0.05 \mathrm{Jy}\right.$, Acke et al. 2012) and ALMA at $870 \mu \mathrm{m}$ (taken as a $3 \sigma$ upper limit: $F_{850}<4.8 \mathrm{mJy}$, Boley et al. 2012); their spatial location is constrained by the instruments point spread functions/beam to be smaller than $\sim 20$ AU. Additional Spitzer-IRS spectroscopic data is available: a small excess emission ( $\lesssim 1 \mathrm{Jy}$ ) shortward of $30 \mu \mathrm{m}$ 
Table 2. Properties of the Fomalhaut exozodiacal disk derived from the fit to the data.

\begin{tabular}{|c|c|c|c|c|}
\hline \multirow[t]{2}{*}{ Properties } & \multicolumn{2}{|c|}{ Hot ring } & \multicolumn{2}{|c|}{ Warm belt } \\
\hline & Approach 1 & Approach 2 & Approach 1 & Approach 2 \\
\hline \multirow{2}{*}{ Density peak $r_{0}[\mathrm{AU}]$} & $0.09^{*}\left(0.23^{1}\right)$ & $0.09^{*}\left(0.23^{1}\right)$ & 1.59 & 2.52 \\
\hline & & & {$[1.62,2.43]$} & {$[1.54,2.15]$} \\
\hline \multirow[t]{2}{*}{ Inner density slope $\alpha_{\text {in }}$} & $+10^{*}$ & $+10^{*}$ & $+10^{*}$ & +3 \\
\hline & & & & {$[+2.99,+7.52]$} \\
\hline \multirow{2}{*}{ Outer density slope $\alpha_{\text {out }}$} & $-6^{*}$ & $-6^{*}$ & -1.0 & $-1.5^{*}$ \\
\hline & & & {$[-3.62,-0.60]$} & \\
\hline \multirow{2}{*}{ Mass up to $1 \mathrm{~mm}\left[M_{\oplus}\right]$} & $2.5 \times 10^{-10}$ & $2.6 \times 10^{-10}$ & $2.86 \times 10^{-6}$ & $1.87 \times 10^{-6}$ \\
\hline & & & {$\left[6.12 \times 10^{-7}, 9.53 \times 10^{-5}\right]$} & {$\left[1.14 \times 10^{-6}, 7.81 \times 10^{-5}\right]$} \\
\hline Maximum surface density $\left[\mathrm{cm}^{-2}\right]$ & $7.24 \times 10^{11}$ & $7.46 \times 10^{11}$ & 110 & 66.6 \\
\hline Maximum $V$-band optical depth $\tau_{\perp}{ }^{3}$ & $2.2 \times 10^{-3}$ & $2.3 \times 10^{-3}$ & $9.8 \times 10^{-5}$ & $1.2 \times 10^{-4}$ \\
\hline Fractional luminosity $L_{\mathrm{D}} / L_{\star}$ & $5.89 \times 10^{-3}$ & $5.71 \times 10^{-3}$ & $5.04 \times 10^{-4}$ & $6.09 \times 10^{-4}$ \\
\hline Composition & $\mathrm{C}$ & $\mathrm{C}$ & $\mathrm{Si}+\mathrm{C}$ & $\mathrm{Si}+\mathrm{C}$ \\
\hline \multirow{2}{*}{ Minimum grain size $[\mu \mathrm{m}]$} & 0.01 & 0.01 & 3.51 & 2.31 \\
\hline & & & {$[1.37,56.1]$} & {$[0.99,57.2]$} \\
\hline \multirow{2}{*}{ Grain size slope } & -6 & -6 & -4.8 & -4.06 \\
\hline & & & {$[-6.0,-3.8]$} & {$[-6.0,-3.7]$} \\
\hline Typical temperatures $[\mathrm{K}]^{2}$ & {$[2000,2200]$} & {$[2000,2200]$} & {$[403,464]$} & {$[326,364]$} \\
\hline$\chi^{2}$ (d.o.f. $\left.=30\right)$ & - & - & 46.9 & 47.9 \\
\hline
\end{tabular}

Notes. Approach 1 labels the model with free outer slope, approach 2 that with free inner slope. Numbers in bold are the parameters of the smallest $\chi^{2}$ fits, while the intervals give the $1 \sigma$ confidence intervals from the Bayesian analysis. The ${ }^{(*)}$ exponent marks the fixed parameters. (1) Approximate density peak position of the smallest grains (they vanish below that distance). (2) Give range of temperatures at $r_{0}$ from the smallest to $1 \mathrm{~mm}$ grains. For the hot ring, the temperature of the smallest grains is given at their sublimation distance. ${ }^{(3)}$ The optical depth is directly proportional to the dust mass and therefore constitute another definition to this free parameter (given in $V$-band by convention).

is reported by Su et al. (2013). Due to large calibration uncertainties in the absolute photometry $(\sim 10 \%)$ compared with the interferometric measurements, we do not attempt to fit this spectrum. The IRS spectrophotometry is represented with $3 \sigma$ upper limits in Fig. 5 to verify the compatibility of the model at these wavelengths.

The GRaTeR code is used to adjust the dataset in several steps. We point out that all the results presented below correspond to thermal emission by the dust and that the scattering of the stellar spectra by the grains is always negligible (although systematically calculated).

As a first step, we assess the hot population, assumed to be composed of compact carbonaceous grains, because they are very small and lie within the silicate sublimation zone (Paper II). We adopt the same grid of models as the one presented in Table 4 of Paper II, yielding 200 000 models for 5 free parameters: the minimum grain size $a_{\min }$, the slope of the size distribution $\kappa$, the surface density peak $r_{0}$, outer slope $\alpha_{\text {out }}$ and the total disk mass $M_{\text {dust }}$ (with the maximum grain size fixed to $1 \mathrm{~mm}$ ). The inner density slope is assumed to be steep $\left(\alpha_{\text {in }}=+10\right)-$ but this has no impact as this region is within the sublimation radii of carbons as we will see.

As a second step we focus on the disk's warm component and take advantage of the linearity of the data that allows us to sum the contributions from the two components. Using the same parameter space, we adjust simultaneously 6 free parameters: the mass, geometry and disk properties of the warm component and the mass of the hot component (modeled in step 1) to the 36 measurements composing the full dataset (VINCI, KIN, MIPS, PACS, ALMA). The warm ring is made of a 50-50 mixture of astronomical silicates and carbonaceous material characteristic of the material properties commonly inferred from debris disks and solar system asteroids studies. We do not vary this parameter as it is found to be essentially unconstrained by the observations in the previous study.

Two approaches are used. In the first approach, the outer slope of the density profile is taken as a free parameter, and the inner slope is assumed to be very steep (fixed to +10 ). In the second approach, the outer slope is fixed to $-1.5-$ close to the best-fit for the first approach and consistent with the profile expected for a collisional equilibrium under the effect of sizedependent radiation pressure (Thébault \& Wu 2008) - and we vary the inner slope $\alpha_{\text {in }}$.

A $\chi^{2}$ minimization is performed as well as a Bayesian statistical analysis to measure the likelihood of the model parameters. The best-fitting parameters and Bayesian estimates discussed in this section are summarized in Table 2 and the resulting models are shown in Figs. 5 and 6. Probability curves are shown in Appendix B.

\subsection{The hot population}

As expected, the hot component is mostly constrained by the near-infrared data and best explained by a very narrow ring of $\gtrsim 10 \mathrm{~nm}$ refractive grains, with a density profile peak matching the sublimation distance of carbons $\left(r_{0}=0.09 \mathrm{AU}\right)$, where only the largest grains survive. The best model $\left(\chi_{r}^{2}=1.4\right.$ with 20 degrees of freedom, d.o.f.) is found for a minimum grain size matching the smallest values of the parameter space $\left(a_{\min }=\right.$ $10 \mathrm{~nm}, \kappa=-6)$, although the Bayesian analysis favors $a_{\min }=$ $20 \mathrm{~nm}$. In fact, the exact properties of this ring have little impact on the resulting SED, as long as the grain size-dependent temperatures and total mass are consistent with the $K$-band excess. The slopes of the density profile and of the size distribution 
are qualitatively very steep but their exact values are not well identified due to the strong dependence of temperature with distance and grain size. However, the data also carries spatial information. In particular the dust must be confined within the (Gaussian) field-of-view of the VLTI and it must not let too much emission through the complex KIN transmission map in the mid-infrared. Grains larger than $\sim \lambda / 2 \pi \simeq 0.3 \mu \mathrm{m}$ are also inefficient emitters in the $K$-band, and due to the single power law used, very small grains can become dominant. For these reasons and despite the modeling degeneracy between grain size and disk location, the minimum grain size cannot exceed a few $\sim 10 \mathrm{~nm}$. For instance, the best model found with $a_{\min }=1 \mu \mathrm{m}$ has a $\chi_{\mathrm{r}}^{2}$ of 2.9. This result is reminiscent of what was found by Defrère et al. (2011a) who show that the exozodi of Vega must be composed of grain much smaller than $1 \mu \mathrm{m}$ based on multiwavelengths constraints in the near-IR. We use $a_{\min }=10 \mathrm{~nm}$ as a working assumption for the rest of the study although what really matters is to determine which of the grains are the emitters.

In the inner solar system, the dust probed by the NASA's deep Impact mission likely consists of $\sim 20 \mu \mathrm{m}$-sized highly porous dust aggregates (e.g. Kobayashi et al. 2013). The hot exozodi of Fomalhaut rather consists of nanometer to submicrometer grains that we interpret as the elemental monomers produced after the break-up of larger dust particles. As demonstrated in Paper II (Fig. 6 and Table 5), models with higher porosity provide less good fit to the data. For instance, setting the porosity to $85 \%$ yields a smallest $\chi_{\mathrm{r}}^{2}$ of 1.8 for $a_{\min }=1.5 \mu \mathrm{m}$. A large amount of sensitive measurements would be needed to go beyond this solid carbon grain model (see e.g. Lebreton et al. 2012).

The new sublimation prescription provides a more reliable estimate of the dust location with respect to the constant sublimation temperature assumption (Fig. 7). With $\alpha_{\text {out }}=-6$ (i.e. a very narrow ring), the sublimation distance of $0.01 \mu \mathrm{m}$ grains is $0.235 \mathrm{AU}\left(\simeq d_{2}\right)$; for these grains the emission falls down to $10 \%$ of the peak flux at 0.34 AU. Grains larger than $a_{\min }$, in particular those in the range $0.1-0.5 \mu \mathrm{m}$ that lie close to $d_{1}$ contribute to thermal emission in similar proportions compared with $10 \mathrm{~nm}$ grains. Thus, independent from our choices for the parameter space limits, the emission is by far dominated by unbound grains and a robust result is that this hot exozodi is composed of grains smaller than $\sim 0.5 \mu \mathrm{m}$. The dust mass is dominated by the smallest grains, due to $\kappa<-4$. We would like to stress that forcing the material sublimation temperatures to higher values would not yield better fit to the data as it would only move the peak emission toward even shorter wavelengths (Table 2, Fig. 5). In the following, we adopt the above parameters for the hot ring and keep the total dust mass as the single free parameter for this component (Table 2).

\subsection{The warm population}

With the first approach (variable outer slope, fixed inner slope), the analysis indicates that the mid- to far-infrared data is best fitted by a dust belt peaking at $r_{0}=1.6 \mathrm{AU}$ and declining slowly as $r^{-1}$. The minimum size is close to the blowout limit $\left(a_{\text {blow }}=8.8 \mu \mathrm{m}\right)$, in a rather steep distribution $(\kappa<-3.8)$, yielding a reduced $\chi^{2}$ of 1.56 (with 30 d.o.f.). These properties are consistent with the theoretical expectations that the warm population is produced through a collisional cascade in a parent-body belt, although the steep distribution might be more indicative of a recent catastrophic collision than a steady-state debris disk.

Interestingly, the second approach (fixed outer slope, variable inner slope) yields similar results to those of the first one,
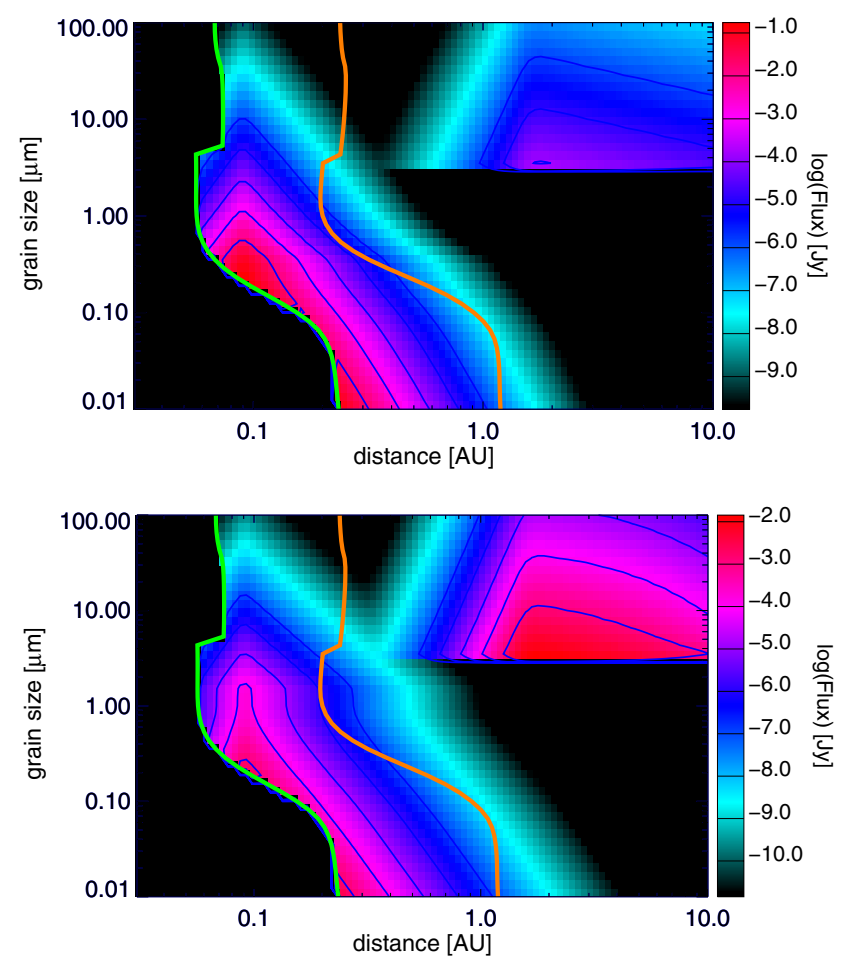

Fig. 7. Maps showing the absolute distribution of flux as a function of distance from the star and grain size. Top panel: $\lambda=2.18 \mu \mathrm{m}$, bottom panel: $\lambda=12.0 \mu \mathrm{m}$. The orange and green lines mark the sublimation distances of silicate and carbon grains respectively.

except that a second family of solutions arises, with very small grains located at the outer edge of the range of explored peak radii. Nonetheless this solution can be excluded based on the absence of silicate features in the Spitzer/IRS spectrum that would be created by such tiny silicate grains. We add prior information in the Bayesian analysis to reject solutions with $a_{\text {min }}<a_{\text {blow }} / 10$ (Appendix B.2) and find a best reduced $\chi^{2}$ of 1.60 . Due to the different geometrical profile assumed, the peak radius is found to lie further out, at $\sim 2.5 \mathrm{AU}$. The inner ring is not as steep as previously assumed $\left(\alpha_{\text {in }}=+3\right)$, which is suggestive of an inward transport of material by Poynting-Robertson drag mitigated by destructive collisions (see for example Löhne et al. 2012).

\section{5. modeling summary}

Figure 5 shows an excellent fit to the spectral energy distribution. The near-infrared excess is almost solely produced by the hot ring at the sublimation distance. The warm belt at $2 \mathrm{AU}$ is sufficient to explain the measured SED from mid- to far-infrared wavelengths and it is compatible with the Spitzer/IRS excesses. Furthermore the null excesses are mostly produced by the hot component below $\sim 10 \mu \mathrm{m}$ in the 2007 configuration, but their rising profiles at longer wavelengths requires the contribution from the warm component. All the model excesses are compatible with the measurements within $\sim 1 \sigma$ except for the short equivalent baseline in the 2008 sample that is underpredicted by $\sim 2 \sigma$ upon $11 \mu \mathrm{m}$. A possible interpretation would be the existence of an azimuthal asymmetry in the disk. Overall the warm ring is much more massive than the hot ring with respective values of $\sim 2 \times 10^{-6} M_{\oplus}$ and $\sim 2.5 \times 10^{-10} M_{\oplus}$ in grains smaller than $1 \mathrm{~mm}$, but the hot ring is significantly brighter (Fig. 7) due not only to its high temperature, but also to the nature of its constituent grains. The tiny grains that the hot ring is made of 

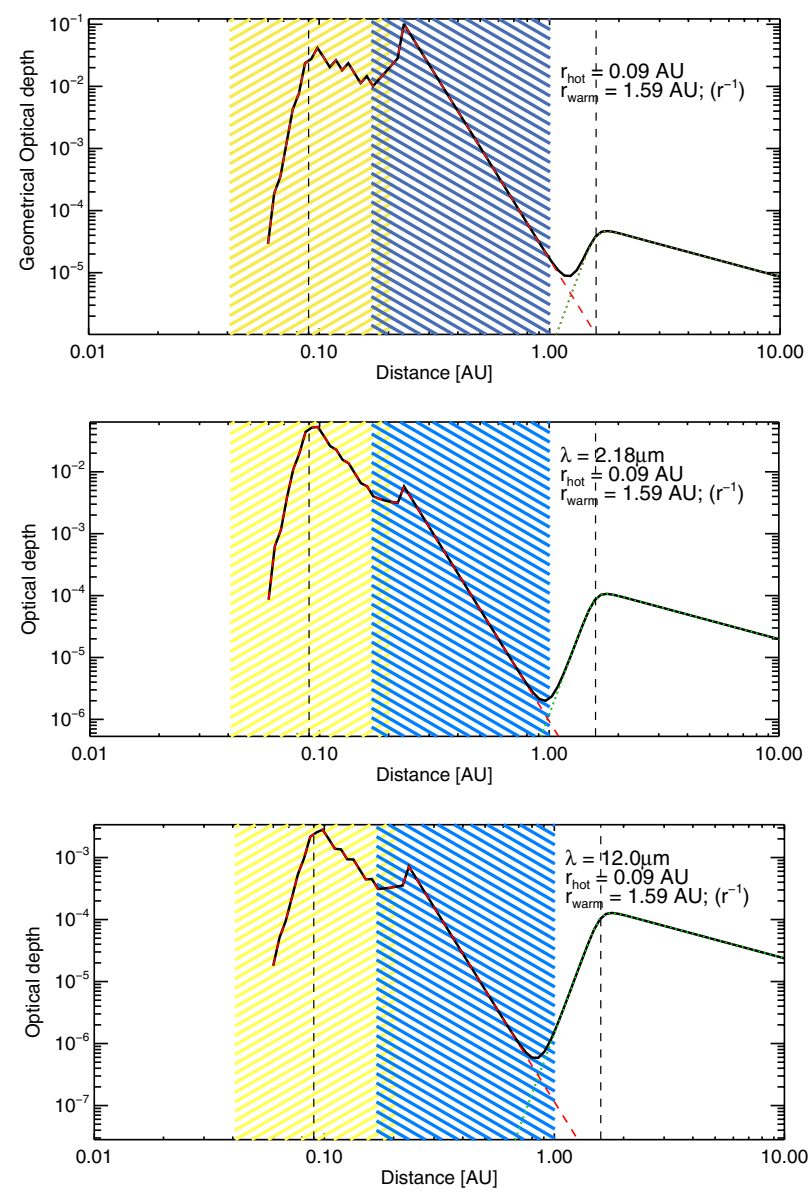

Fig. 8. Radial profiles for the best-fitting double-ring model (approach 1. Red: hot ring; green: warm belt): geometrical vertical depth profiles (top panel), optical depth profiles at $\lambda=2.18 \mu \mathrm{m}$ (middle panel) and $\lambda=12 \mu \mathrm{m}$ (bottom panel). The dashed regions represent the sublimation zones of silicates (blue region) and carbons (yellow). The effect of size-dependent sublimation is evidenced by the ragged profile of the hot ring optical depth. A logarithmic sampling is used with 56 sizes ranging from 0.01 to $1000 \mu \mathrm{m}$.

constitute a much larger effective cross-section than an equivalent mass of material concentrated in large grains. The resulting profiles are illustrated in Fig. 8 that shows the geometrical vertical optical depth and effective vertical optical depths at two representative wavelengths.

In summary, the hot ring at a fraction of AU consists essentially in grains that seem to contradict the dynamical/collisional theoretical constraints ( $a \ll a_{\text {blow }}, \kappa \leq-5.0$, steep density profile). The "warm belt" at about 2 AU seems compatible with the "classical" debris disk picture $\left(a_{\min }, \kappa\right.$ and $\alpha_{\text {out }}$ are compatible with blowout of small grains and a collisional size distribution).

\section{Origin of the dust}

In this section, we test several mechanisms as possible explanations for the peculiar morphology suggested by the modeling results. First, we discuss the viability of parent belts at the locations of the hot ring and the warm belt (Sect. 5.1). Subsequently, we review possible replenishing mechanisms for the warm belt (Sect. 5.2), and test whether the pile-up of dust in the sublimation zone can explain the hot ring (Sect. 5.3). In Sect. 5.4, we examine whether the observed population of unbound grains can be produced by the disruption of larger bodies due to sublimation. Finally, we investigate the role of gas in retaining the small grains in the hot ring by slowing down both their blowout and sublimation (Sect. 5.5). A short summary of our findings is presented in Sect. 5.6.

To compare theory with observation, we approximate the fractional luminosity of dust at radial distance $r$ as the fraction of the star covered by dust at that location:

$\frac{L_{\mathrm{D}}}{L_{\star}}(r) \approx \frac{\sigma_{\mathrm{D}}(r)}{4 \pi r^{2}}$.

Here, $\sigma_{\mathrm{D}}(r)$ is the collective cross section of the dust at radial distance $r$. When the excess flux can be assumed to be entirely due to spherical grains of a single size $a$ (and hence with a mass of $\left.m=4 \pi \rho_{\mathrm{d}} a^{3} / 3\right)$, the fractional luminosity is related to the total dust mass according to

$\frac{L_{\mathrm{D}}}{L_{\star}}(r) \approx \frac{3 M_{\mathrm{D}}}{16 \pi \rho_{\mathrm{d}} a r^{2}}$

\subsection{In-situ dust production through a collisional cascade?}

Excess infrared emission from debris disks is normally interpreted as thermal emission from dust produced through mutual collisions between larger bodies in a planetesimal belt. Therefore, we first test whether the observed NIR excess can be explained by the production of small dust grains by asteroid belts at the locations of the hot ring and the warm belt.

In steady-state collisional evolution, a planetesimal belt at a given radial distance from the star can only contain a maximum amount of mass at any given time, because more massive belts process their material faster (Dominik \& Decin 2003; Wyatt et al. 2007a). Assuming that the size distribution follows the classical Dohnanyi (1969) power law $(\kappa=-3.5$, valid if the critical specific energy for dispersal $Q_{D}^{\star}$ is independent of particle size) at all sizes, and extends down to the blowout size, the mass corresponds to a maximum fractional luminosity of (Wyatt et al. 2007a)

$$
\begin{aligned}
\max \left[\frac{L_{\mathrm{D}}}{L_{\star}}\right]= & 7.0 \times 10^{-9}\left(\frac{r}{1 \mathrm{AU}}\right)^{7 / 3} \\
& \times\left(\frac{\mathrm{d} r / r}{0.5}\right)\left(\frac{a_{\mathrm{c}}}{30 \mathrm{~km}}\right)^{0.5}\left(\frac{Q_{\mathrm{D}}^{\star}}{150 \mathrm{~J} \mathrm{~kg}^{-1}}\right)^{5 / 6}\left(\frac{e}{0.05}\right)^{-5 / 3} \\
& \times\left(\frac{M_{\star}}{1.92 M_{\odot}}\right)^{-5 / 6}\left(\frac{L_{\star}}{16.63 L_{\odot}}\right)^{-5 / 6}\left(\frac{t_{\mathrm{age}}}{440 \mathrm{Myr}}\right)^{-1}
\end{aligned}
$$

Here, we inserted fiducial values for the relative width of the planetesimal belt $\mathrm{d} r / r$, the radius of the largest bodies $a_{\mathrm{c}}$, the critical specific energy for dispersal $Q_{\mathrm{D}}^{\star}$, and the mean planetesimal eccentricity $e^{4}$. For the stellar mass $M_{\star}$, the stellar luminosity $L_{\star}$, and the age of the system $t_{\text {age }}$, we used the parameters of Fomalhaut found by Mamajek (2012). Cratering collisions, which have a specific energy lower than $Q_{\mathrm{D}}^{\star}$, lead to an increased erosion of large bodies, and therefore a faster processing of the available material, and a lower maximum fractional luminosity at any given age. However, they are not accounted for in the model of Wyatt et al. (2007a). Including cratering collisions would lower the numerical factor in Eq. (27) by about a factor 4 to 5 (Kobayashi \& Tanaka 2010). Our choice of $Q_{\mathrm{D}}^{\star}=150 \mathrm{~J} \mathrm{~kg}^{-1}$ can be seen as a conservative estimate.

4 These values were found to give a good fit to debris disks around a
sample of A stars (Wyatt et al. 2007b), and can be used as first order
estimates for these poorly constrained parameters in the case of exozodiacal dust. 
Evaluating Eq. (27) at the radial locations of the hot ring $(r \approx 0.25 \mathrm{AU})$ and the warm belt $(r \approx 2 \mathrm{AU})$, gives maximum fractional luminosities of $2.7 \times 10^{-10}$ and $3.5 \times 10^{-8}$, respectively. We note that the modeling results indicate steeper size distributions $(\kappa<-4.0)$ for both components, which would yield much higher maximum fractional luminosities (using Eq. (16) of Wyatt et al. 2007a). However, the observations only probe the lower end of the size distribution, and it is unlikely that the steep power law extends all the way to parent body sizes. In contrast, the size distribution is expected to be shallower at large sizes, where the strengthening of bodies due to self-gravity becomes important. Furthermore, the theoretical $\kappa=-3.5$ is confirmed observationally for km-sized objects in the solar system's asteroid belt (Dohnanyi 1969). In a more thorough discussion of the stringentness of the maximum fractional luminosity, Wyatt et al. (2007a) find that by pushing the parameters, the fractional luminosity can be made to exceed the maximum given by Eq. (27) by a factor 100 at most. Since the fractional luminosities derived from modeling the interferometric data (Table 2) are more than two orders of magnitude higher than the maximum ones, we conclude that neither of the two components can be explained by in-situ asteroid belts that have been in collisional equilibrium for the age of the system.

\subsection{Replenishing the warm belt}

If the warm belt is in steady state (and not a transient phenomenon), some mechanism must operate to replenish the observed dust, other than an in-situ collisional cascade. A possible source of the material is the outer cold belt at about $140 \mathrm{AU}$. We now proceed to estimate the rate at which the dust needs to be replenished, and then examine whether PR drag from the cold belt is capable of providing this mass flux.

The warm belt is found to have a relatively steep size distribution $(\kappa<-4.0)$, and therefore its total dust mass is dominated by the smallest grains present. In the warm belt these are particles close to the blowout size, which are destroyed by collisions on a timescale of several thousands of years (Eq. (21)). With a dust mass of a few times $10^{-6} M_{\oplus}$, the mass flux through the warm belt must be of the order of $10^{-9} M_{\oplus} \mathrm{yr}^{-1}$.

PR drag can only supply a limited amount of material, since grains undergo mutual collisions as they migrate inwards, and the fragments produced in these collisions can be blown out. This was demonstrated by Wyatt (2005), using a model that assumes a single particle size, fully destructive collisions, and circular orbits ${ }^{5}$. Based on this model, we can estimate the collisionlimited PR drag mass flux from a dust source located at $r_{\text {source }}$ inward to a radial distance $r$, which is

$\max \left[\dot{M}_{\mathrm{PR}}(r)\right]=\frac{L_{\star} \sqrt{G M_{\star}} \beta Q_{\mathrm{pr}}}{2 c^{3}\left(\sqrt{r_{\text {source }}}-\sqrt{r}\right)}$.

Here, $L_{\star}$ is the stellar luminosity, $G$ is the gravitational constant, $M_{\star}$ is the stellar mass, $Q_{\mathrm{pr}}$ is the wavelength-averaged radiation pressure coefficient, $c$ is the speed of light. A detailed derivation of this equation will be presented by R. van Lieshout et al. (in prep.). This maximum is independent of the amount of material at the dust source. Also, its dependence on grain properties is through $Q_{\mathrm{pr}}$ and $\beta$, for which we know $0<Q_{\mathrm{pr}}<2$, and $\beta<0.5$ for dust released from large $(\beta \approx 0)$ parent bodies on circular orbits.

\footnotetext{
5 The model of Wyatt (2005) ignores stellar wind drag, but this process is not expected to be important for Fomalhaut and other A stars, because their mass loss rates are predicted to be very low.
}

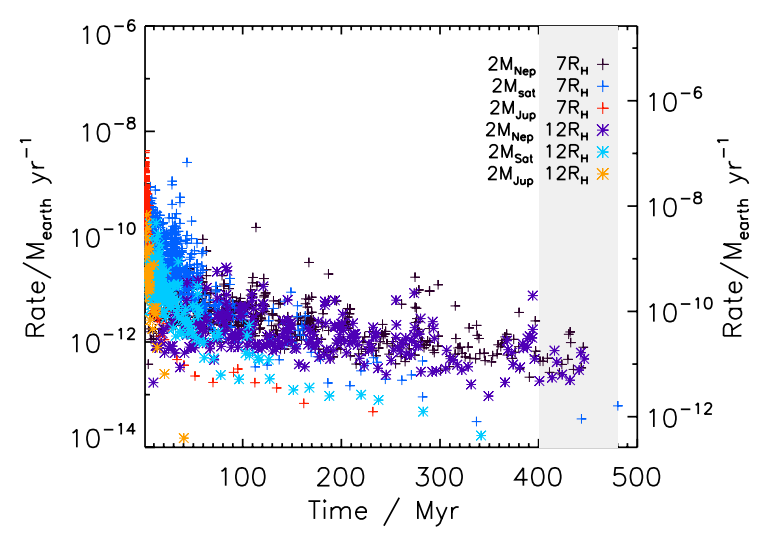

Fig. 9. Steady-state time evolution of the rate at which material is scattered inward of $2 \mathrm{AU}$ through planet scattering, in Earth masses per year; left axis: $M_{\text {outer }}=4 M_{\oplus}$, right axis: $M_{\text {outer }}=105 M_{\oplus}$. Several compact planetary configurations are tested with with 7 or 12 equal mass (2 Jupiter- 2 Neptune- or 2 Saturne-mass planets), equally spaced by 7 or 12 Hill-radii. The grey area represents the estimated age of Fomalhaut.

We evaluate Eq. (28) at the location of the warm belt $(r \approx 2 \mathrm{AU})$, using $r_{\text {source }}=140 \mathrm{AU}$, the stellar parameters of Fomalhaut, $Q_{\mathrm{pr}}=1.0$, and $\beta=0.5$. The $\beta$ ratio is that of the smallest bound grains, which are dragged in the most efficiently, and can therefore provide the highest mass flux. The resulting maximum mass flux is about $1.2 \times 10^{-12} M_{\oplus} \mathrm{yr}^{-1}$, which is several orders of magnitude smaller than the mass flux required to maintain the warm belt. This indicates that PR drag is not the main replenishing mechanism for the warm belt.

Another possible mechanism for transporting material from the outer cold belt to the warm belt is the inward scattering of small bodies by planets (Bonsor \& Wyatt 2012; Bonsor et al. 2012). The outer belt mass is constrained to be between $4 M_{\oplus}$ (Boley et al. 2012) and $110 M_{\oplus}$ (Acke et al. 2012) and the star age is $440 \pm 40 \mathrm{Myr}$ (Mamajek 2012). If a chain of planets were to orbit between the cold and warm belts, these planets could scatter small bodies inwards, in essence resupplying the warm and hot belt with material. Bonsor et al. (2012) used $N$-body simulations to investigate this scattering process and determined a maximum flux of scattered particles as a function of time. This maximum occurs if a chain of tightly packed, low mass planets, were to orbit between the belts. Here, we apply these models to the case of Fomalhaut, scaling the simulations to account for the stellar mass of $2.1 M_{\odot}$ and the inner edge of the outer belt at $133 \mathrm{AU}$. The results are shown in Fig. 9. The important information given by this plot is the maximum possible rate at which material can be scattered inwards, given by the upper envelope of the scattered points. By assuming that the material is efficiently converted to small dust, this also gives us the maximum rate at which the observed small dust in the hot and warm belts could be resupplied. This process could potentially provide a mass flux of up to $5 \times 10^{-11} M_{\oplus} \mathrm{yr}^{-1}$ continuously for the age of the system, which is still not sufficient to compensate the collision rate $\left(4 \times 10^{-10} M_{\oplus} \mathrm{yr}^{-1}\right)$. This value is very much a maximum mass flux, as it was calculated using the planet configuration that is the most efficient at scattering material inwards and it assumes that the scattered objects are entirely disrupted upon entering the inner regions of the Fomalhaut planetary system.

\subsection{Accumulation of sublimating dust grains?}

For the hot ring, the radial distribution of dust exhibits a strong peak in surface density in the carbon sublimation zone, with 
much lower levels of dust further out (see Fig. 8). This spatial profile suggests the existence of a mechanism to confine the carbonaceous dust in the sublimation zone.

Kobayashi et al. (2009) predicted that the dual effect of PR drag and radiation pressure blowout can result in an accumulation of grains around the sublimation radius. In the following we briefly explain the pile-up mechanism in terms of the three stages identified by Kobayashi et al. (2009). (1) Initially, the grains are far away from the star, and sublimation is negligible. The grains spiral inward due to PR drag and gradually heat up as they come closer to the star. (2) As the dust temperature approaches the sublimation temperature, the grains start to lose mass due to sublimation. As a consequence of the increasing cross-section-to-mass ratio of the dust grains, radiation pressure gains in relative importance compared to gravity (i.e. the $\beta$ ratio of the dust grains becomes higher). This increases the eccentricity of the dust orbits, and therefore their orbital size. Hence, the inward radial migration is slowed down. Eventually, the decrease in semi-major axis due to PR drag is compensated by the increase due to sublimation. This happens roughly at the radial distance where the PR drag timescale equals the sublimation timescale (Kobayashi et al. 2008). (3) Finally, the size of the dust grains drops below the blowout radius, and their orbits become unbound. At this point the grains either leave the system, or they fully sublimate before they exit the sublimation zone. The net outcome of this process is an accumulation of dust in the sublimation zone, and this result is very robust against various grain properties (composition, porosity, fractal structure).

The pile-up mechanism is a result of the interplay between PR drag and sublimation, and was investigated for drag dominated disks (i.e. disks in which collisions are unimportant). If collisions are significant, they may inhibit the process of dust pile-up. Furthermore, a significant pile-up requires very low orbital eccentricities $e \lesssim 10^{-2}$ (Kobayashi et al. 2008, 2011). PR drag can circularize the orbits of dust particles, but only if the source region is distant enough. These caveats will be examined in further detail by van Lieshout et al. (in prep.).

The material in the pile-up needs to be replenished from further out by PR drag. Assuming that a dust source is located at the radius of the warm component $\left(r_{\text {source }}=2 \mathrm{AU}\right)$, without specifying how this source can be maintained, we now use Eq. (28) to estimate how much material can be transported inward to the hot ring at $r=0.23 \mathrm{AU}^{6}$. Inserting the parameters of Fomalhaut, together with $Q_{\mathrm{pr}}=1.0$ and $\beta=0.5$, yields a maximum mass flux of $1.4 \times 10^{-11} M_{\oplus} \mathrm{yr}^{-1}$.

If the orbital eccentricities of the dust grains are sufficiently low, a pile-up of dust will occur at roughly the radial distance where the PR drag timescale equals the sublimation timescale. The dust stays in the pile-up until it is completely sublimated, or sublimated to below the blowout size, which approximately takes a sublimation timescale ${ }^{7}$. Therefore, the dust stays in the pile-up for a PR drag timescale.

A rough estimate of the maximum total mass of piled up dust can be found by multiplying the maximum mass flux rate found earlier with the PR drag timescale at the pile-up location.

\footnotetext{
6 In the best fit model, two populations of dust contribute equally to the hot component emission, one at $r=0.1 \mathrm{AU}$, and one at $r=0.23 \mathrm{AU}$. The latter is most optimistic choice for the PR drag scenario, resulting in the highest mass flux.

7 Individual dust grains survive for longer than a sublimation timescale, since they end up on eccentric orbits that take them out of the sublimation zone. However, the time they spend in the pile-up, where they are observed, corresponds to the sublimation timescale.
}

For $r=0.23 \mathrm{AU}$, the PR drag timescale is $22 \mathrm{yr}$, resulting in a maximum dust mass of $3.1 \times 10^{-10} M_{\oplus}$, which is compatible with the modeled hot component mass. However, the maximum fractional luminosity due to this amount of mass in bound grains at this location (Eq. (26)), is only $9.2 \times 10^{-6}$, which is several orders of magnitude lower than the value of the best fit model. This estimate is independent of the amount of material at the dust source, and only depends on grain properties through $\beta$, which should not be higher than $\beta=0.5$. The reason for the discrepancy is that the dust grains found by the model are much smaller than the $\beta=0.5$ particles considered for the pile-up mechanism, and therefore constitute much more cross section for the same amount of mass. Since these small grains are below the blowout size, they are removed from the system on timescales much shorter than the PR drag timescale. Hence, the pile-up of dust alone cannot explain the observed excess emission of the hot component.

\subsection{The release of small dust grains in the sublimation zone}

The observation of dust grains with sizes far below the blowout size presents a problem ${ }^{8}$. These particles have very short survival timescales, and therefore have to be replenished quickly. Their detection in the sublimation zone indicates that they could be released from unseen larger bodies that fall apart as they sublimate. The increase in the number of particles, with conservation of total mass, would lead to an increase in collective cross section, and the steep dependence of sublimation on temperature could explain the sharp peak in emission in the sublimation zone.

Larger bodies could be transported into the sublimation zone by various processes, such as P-R drag, or inward scattering by planets. Alternatively, the small particles could be released by an evaporating planet that is present in the sublimation zone for an extended period and gradually loses material. For now, we ignore what is the exact mechanism that provides the material, but rather focus on the mass source term $\dot{M}$ required to explain the observed fractional luminosity.

The fractional luminosity of the hot ring can be approximated by Eq. (26). This is possible because its size distribution is very steep, so the cross section is dominated by the smallest grains, and because its radial distribution is a sharp spike, so all grains are roughly located at the same radius. Furthermore, because of the steep size distribution $(\kappa<-4.0)$, the total dust mass in the hot ring is dominated by the smallest grains. Hence, the modeling results provide a rough constraint on the product of the mass source term $\dot{M}$ and the survival timescale of the small grains $t_{\text {surv }}$, according to

$\frac{L_{\mathrm{D}}}{L_{\star}}=\frac{3 \dot{M} t_{\text {surv }}}{16 \pi \rho_{\mathrm{d}} a r^{2}}$.

A closer inspection of the modeling results reveals that the flux of the hot ring is mainly due to two populations of dust, which have similar contributions (see Fig. 7). The reason for this complication is that the radial distribution is truncated at the size dependent sublimation radius. One population consists of very small grains $(a=0.01 \mu \mathrm{m})$ located at $r=0.23 \mathrm{AU}$, the other of slightly larger grain $(a=0.2 \mu \mathrm{m})$ at $r=0.10 \mathrm{AU}$. These slightly larger grains are still well below the blowout size. In the following we will test both sets of parameters when evaluating the

\footnotetext{
8 Very small grains sometimes have $\beta$ ratios below unity, due to their low optical efficiencies. However, for the material types tested here, and Fomalhaut as the host star, $\beta$ stays well above unity, and the smallest grains are unbound (see Fig. 2).
} 

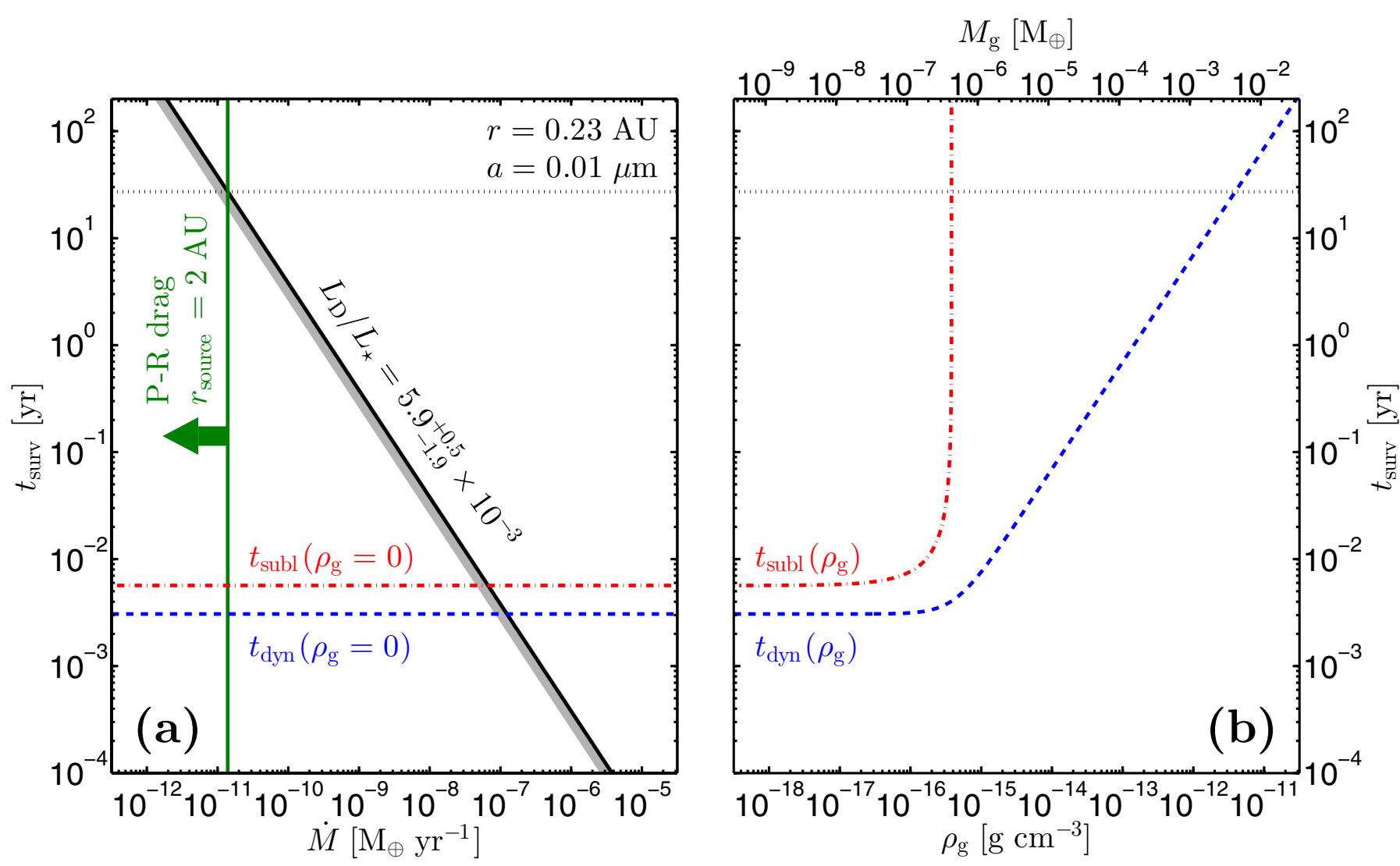

Fig. 10. Summary of the constraints on the hot ring, assuming it consists of $0.01 \mu \mathrm{m}$ carbon grains located at 0.23 AU. Panel a) the diagonal line shows the relation between the mass flux and the dust grain survival timescale, as constrained by the observed fractional luminosity (black, with error margins in grey, Eq. (29)). The horizontal lines indicate the typical timescales (in a gas free environment) for destruction by sublimation (red, dash-dotted, Eq. (30)), and removal by blowout (blue, dashed, Eq. (31)). The vertical line with the arrow indicates the maximum mass flux due to P-R drag from a very dense source region located at $2 \mathrm{AU}$ (green, Eq. (28)). Panel b) the dependence on gas density of the sublimation timescale (red, dash-dotted, Eq. (30)), and the blowout timescale (blue, dashed, the sum in quadrature of Eqs. (31) and (32)) The top axis gives the total gas mass corresponding to the midplane gas densities on the bottom axis, assuming the gas is located in a vertically isothermal ring of width $\Delta r=r$ (Eq. (34)). The horizontal, black, dotted line marks the minimum survival time required if the observed material is to be provided by PR drag. It extends across both panels to show the gas density and total gas mass this would imply.

survival timescale. For the material density we assume that of carbon with a porosity of $5 \%\left(\rho_{\mathrm{d}}=1.85 \mathrm{~g} \mathrm{~cm}^{-3}\right)$.

We now make an estimation of the survival timescale of the relevant dust grains, in order to find the required mass production or influx to explain the observed fractional luminosity. The mechanisms that are critical to the survival of small grains close to a star are sublimation and blowout. Sublimation destroys the grains on a timescale of

$t_{\text {subl }}=\frac{a}{|\dot{a}|}$,

where $\dot{a}$ is given by Eq. (17). This is the time it takes for a grain to sublimate, assuming the sublimation rate remains constant. $t_{\text {subl }}$ is highly dependent on $r$ (through dust temperature), and therefore uncertain. For the blowout timescale, we take

$t_{\mathrm{dyn}}=\sqrt{\frac{5 r^{3}}{2 G M_{\star}(\beta-1)}}$,

which is the time it takes a particle to move outward from its release point to twice that radial distance, valid for $\beta \gg 1$ (a derivation is presented in Appendix A.1).

Figures $10 \mathrm{a}$ and $11 \mathrm{a}$ show the constraints that the observations put on the product of $\dot{M}$ and $t_{\text {surv }}$ for the $a=0.01 \mu \mathrm{m}$ grains at $r=0.23 \mathrm{AU}$ and the $a=0.2 \mu \mathrm{m}$ grains at $r=0.10 \mathrm{AU}$, respectively. Also shown are the typical survival timescales of these dust grains, and the maximum mass influx due to PR drag from a source region at $2 \mathrm{AU}$. The figures reveal that a mass source term of at least $\sim 10^{-7} M_{\oplus} \mathrm{yr}^{-1}$ is required to explain the observations, if the grains survive for $t_{\mathrm{dyn}}$. Comparing this with the maximum mass influx due to PR drag indicates that this mechanism cannot provide enough material.

Other processes that release small dust particles in the sublimation zone may yield higher mass source terms. For instance, Rappaport et al. (2012) report a mass loss rate from the possible evaporating planet KIC $12557548 \mathrm{~b}$ of $\sim 10^{-9} M_{\oplus} \mathrm{yr}^{-1}$, sustainable for $\sim 0.2$ Gyr. This rate would still be insufficient to explain Fomalhaut's hot ring, and the dust morphology inferred for KIC 12557548 b is very different from a ring. However, the actual mass loss rate of an evaporating planet is highly dependent on variables such as its mass, size, temperature, and composition.

Alternatively, the estimates of the dust survival timescale could be too low. If the small dust grains would somehow be retained in the sublimation zone, their survival timescale could be larger. One possibility is that the assumption that the disk is completely gas free is not valid in the sublimation zone. In the next subsection, we explore the influence of gas on the dust survival timescales. 

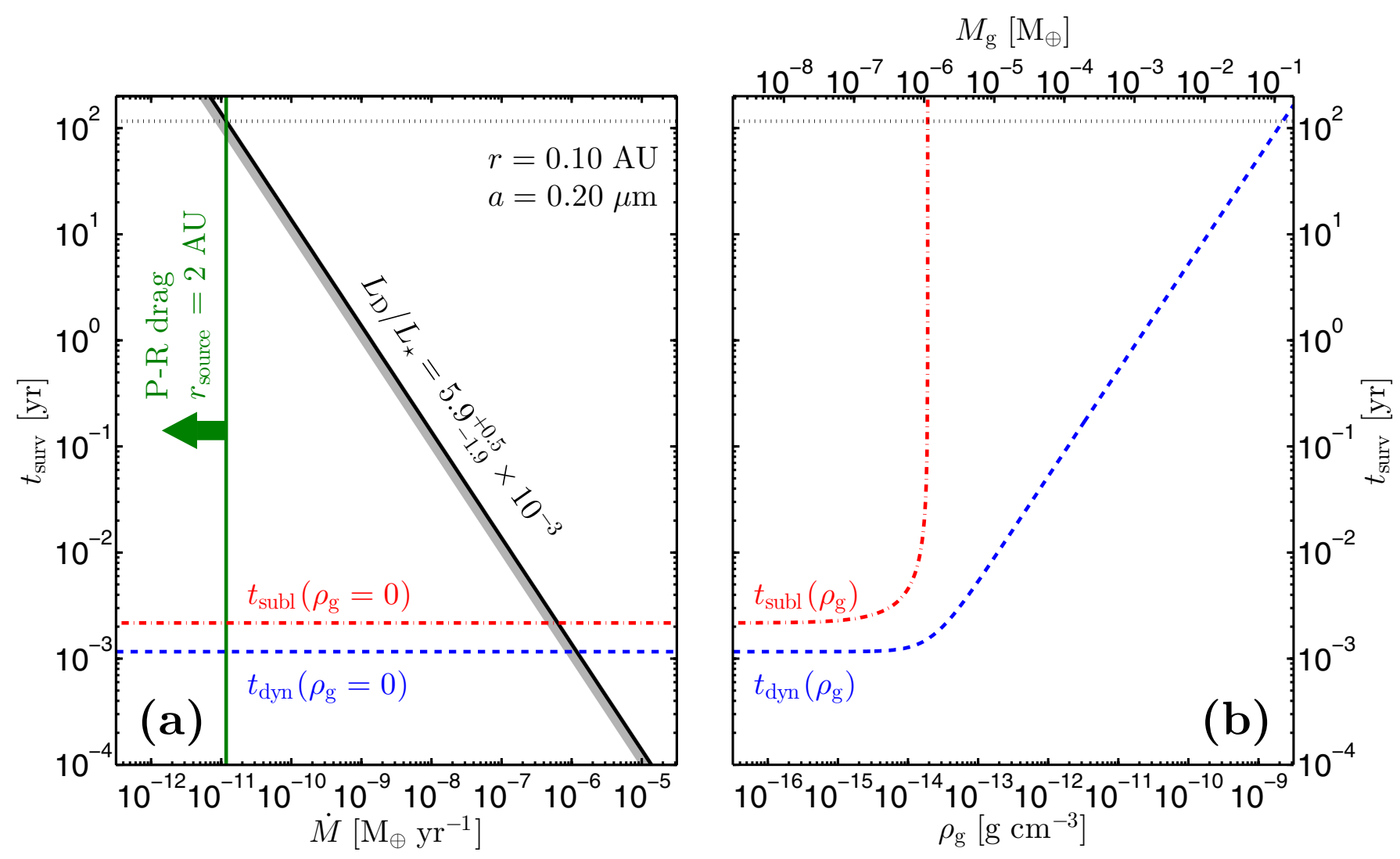

Fig. 11. Same as Fig. 10, but assuming the hot ring consists of $0.2 \mu \mathrm{m}$ carbon grains at $0.1 \mathrm{AU}$. Different scales are used on the horizontal axes in panel b).

\subsection{The influence of gas on dust survival timescales}

Because dust sublimation converts solid dust to gas, some gas is expected to be present in the sublimation zone. The presence of gas increases the blowout timescale, because particles on their way out are slowed down by gas drag. Assuming that the subsonic Epstein drag law can be used for the gas drag force on the small dust grains, we find that the blowout timescale at high gas densities is given by

$t_{\mathrm{dyn}}\left(\Delta r, \rho_{\mathrm{g}}\right)=\frac{\rho_{\mathrm{g}} v_{\mathrm{th}} \Delta r r^{2}}{G M_{\star}(\beta-1) \rho_{\mathrm{d}} S}$.

Here, $\rho_{\mathrm{g}}$ is the mass density of the gas, $v_{\mathrm{th}}$ is the mean thermal speed of the gas, and $\Delta r$ is the distance to be travelled by the blowout grain (this equation is derived in Appendix A.2). This timescale is shown for $\Delta r=r$ in Figs. 10b and 11b, using the gas densities on the lower axes.

By assuming that the gas is confined to a ring around the star with a radial width of $\Delta r$, and that it is vertically isothermal, we can express $t_{\text {dyn }}$ in terms of the total gas mass, and eliminate the dependence on $\Delta r$ and $v_{\text {th }}$. The gas surface density of a vertically isothermal disk with midplane density $\rho_{\mathrm{g}}$ is given by

$\Sigma_{\mathrm{g}}=\frac{\pi \sqrt{\gamma}}{2} \rho_{\mathrm{g}} v_{\text {th }} \sqrt{\frac{r^{3}}{G M_{\star}}}$,

where $\gamma$ is the adiabatic index, for which we assume $\gamma=1.5$. Then, the total gas mass is given by

$$
\begin{aligned}
M_{\mathrm{g}} & =2 \pi r \times \Delta r \times \Sigma_{\mathrm{g}} \\
& =\pi^{2} \sqrt{\gamma} \rho_{\mathrm{g}} v_{\mathrm{th}} \Delta r \sqrt{\frac{r^{5}}{G M_{\star}}},
\end{aligned}
$$

and the blowout timescale becomes

$$
t_{\mathrm{dyn}}\left(M_{\mathrm{g}}\right)=\frac{M_{\mathrm{g}}}{\pi^{2} \sqrt{\gamma G M_{\star} r}(\beta-1) \rho_{\mathrm{d}} s} .
$$

This is shown in Figs. $10 \mathrm{~b}$ and $11 \mathrm{~b}$ with the gas masses on the upper axes.

In the optimistic case that the hot ring is due to $a=0.01 \mu \mathrm{m}$ grains at $r=0.23 \mathrm{AU}$, the hypothesis that the dust is supplied by PR drag from the warm belt requires a survival timescale of about $30 \mathrm{yr}$ (the dotted black line in Fig. 10). This timescale is reached at a gas density of approximately $4 \times 10^{-12} \mathrm{~g} \mathrm{~cm}^{-3}$, which corresponds to a total gas mass of about $5 \times 10^{-3} M_{\oplus}$. Assuming that all this gas is provided by sublimating dust at the maximum PR drag rate, this mechanism needs to have operated for approximately $M_{\mathrm{g}} / \max \left[\dot{M}_{\mathrm{PR}}\right] \approx 5 \times 10^{-3} M_{\oplus} / 1.4 \times$ $10^{-11} M_{\oplus} \mathrm{yr}^{-1} \approx 0.4 \mathrm{Gyr}$, which equals the age of the system, to explain the total gas mass.

The gas will also affect the sublimation of dust grains, since the presence of gas raises the sublimation temperature (see Sect. 3). Figures $10 \mathrm{~b}$ and $11 \mathrm{~b}$ also show the dependence of the sublimation timescale on gas density. This assumes that the ambient gas only consists of carbon, so the gas densities on the lower axes correspond to the partial densities used to compute the sublimation rate in Sect. 3. For high gas densities, the dust particles will not sublimate, but grow. This would stabilize the dust grains, possibly explaining their very high temperatures.

\subsection{Summary of theoretical findings}

The distribution of dust in the inner few AU of the Fomalhaut system that was found by modeling the observations, is difficult 
Table 3. Mass fluxes of several potential supply or production mechanisms, and of the main destruction mechanisms (destructive collisions and radiative transfer blowout).

\begin{tabular}{lc}
\hline \hline Mechanism & Mass flux $\left(M_{\oplus} \mathrm{yr}^{-1}\right)$ \\
\hline \multicolumn{2}{c}{ Warm belt } \\
PR drag from 140 AU & $<1.2 \times 10^{-12}$ \\
Planetesimal scattering & $\lesssim 5 \times 10^{-11}$ \\
Collisions & $\sim-4 \times 10^{-10}$ \\
\hline \multicolumn{2}{c}{ Hot ring } \\
PR drag from 2 AU & $<1.4 \times 10^{-11}$ \\
Evaporating planet ${ }^{1}$ & $\sim 10^{-9}$ \\
Blowout & $\sim-8 \times 10^{-8}$ \\
\hline
\end{tabular}

Notes. ${ }^{(1)}$ This is the value found by Rappaport et al. (2012) for KIC 12557548 b. It is given here for comparison.

to reconcile with a steady-state model. Both the hot and warm components contain more dust than can be explained by in-situ production via a collisional cascade that has operated for the age of the system. If these two components are in steady state (as opposed to being transient phenomena), other processes must be operating to maintain the observed dust populations. We considered several mechanisms as potential sources of the dust. Table 3 gives an overview of the mass fluxes that can be attained by these mechanisms.

The warm belt is dominated by barely bound grains that are primarily destroyed by mutual collisions. It needs a mass flux of the order of $10^{-9} M_{\oplus} \mathrm{yr}^{-1}$ in order to be sustained. This rate cannot be maintained through PR drag of material from the outer cold belt at about 140 AU, but the inward scattering of small bodies by a chain of planets can marginally provide the required mass flux.

The hot ring seems to require an even higher replenishment rate $\left(\sim 10^{-7} M_{\oplus} \mathrm{yr}^{-1}\right)$. It consists of small particles that are removed from the system by blowout. A lower mass flux is possible if these grains are somehow retained near their production site, lengthening their survival timescale. The pile-up of dust due to the interplay of PR drag and sublimation is insufficient to explain the observations. PR drag of dust grains from the warm belt could provide the required mass flux, if a large amount of gas is present in the sublimation zone, which slows down the blowout of unbound grains. The viability of such a substantial gas ring and its consistency with existing observations remain to be tested. Both oxygen and carbon will remain unaffected by radiation pressure around Fomalhaut. In the case of the $\beta$ Pictoris debris disk, Fernández et al. (2006) show that several species can potentially act as self-braking agents on the gas disk, and that more than $0.03 M_{\oplus}$ of gas could be retained consistent with observed upper limits on the column densities.

\section{Discussion}

In the last section, we showed that the hot exozodi of Fomalhaut could be the result of an accumulation of small unbound grains at the carbon sublimation distance brought there by the PR drag effect. For this mechanism to work, a trapping mechanism such as braking by a gaseous component needs to be invoked. Alternative sources of continuum emission, such as free-free emission from a stellar wind, mass-loss events or hot gas, have been discussed e.g. in Paper II, Absil et al. (2008) and Defrère et al. (2012) and can be considered unsatisfying explanations: emission by very hot dust is the most convincing explanation to date. In the solar system, nanometer-sized particles are detected with the STEREO and Ulysse spacecrafts (Meyer-Vernet et al. 2009; Krüger et al. 2010, respectively), with an increase in the particle flux in the inner solar system. These very small grains, which are affected by the Lorentz force (Czechowski \& Mann 2010), could thus be expected in exozodiacal disks. In fact, magnetic trapping of nanograins could be a valuable alternative to the gas-braking mechanisms we have investigated in this study as discussed by Su et al. (2013). We note that it cannot be excluded that these nanograins might produce non-thermal emission, such as a PAH continuum.

The bright hot component can be seen as the "tip of the iceberg" in the sense that it may be a bright counterpart to the warm belt. Our model of the warm belt confirms previous attempts to constrain its properties based on unresolved observations, although our detailed treatment of grain optics and the addition of spatial constraints point towards a closer in location than previously suspected. In particular using a blackbody model, Su et al. (2013) estimated the belt location to be around 11 AU. While preserving the consistency with their dataset, the small FOV of the nulling interferometer impose the warm dust peak distance to be in the $[1.5,2.5]$ AU range after an appropriate subtraction of the hot dust contribution.

Furthermore, future detection of a polarization signal could provide a confirmation of the model, and additional constraints on the grain properties and the disk geometry (in particular its inclination, assumed to match that of the cold belt in this study). We use the MCFOST radiative transfer code (Pinte et al. 2006, 2009) with identical model parameters and assumptions as those discussed above ${ }^{9}$ in order to predict polarimetric signals for the exozodi. We find that the linear polarization integrated over the disk range from $3 \times 10^{-7}$ to $5 \times 10^{-7}$ for the warm belt, and reaches $2 \times 10^{-5}$ to $3 \times 10^{-6}$ for the hot ring in bands $U$ to $I$. These values are compatible with the upper limits of $9 \times 10^{-3}$ to $3 \times 10^{-3}$ given by Chavero et al. (2006). For the hot ring, such signals would potentially be detectable by future sensitive polarimeters.

The warm belt has a distribution of temperatures that ranges from $\sim 320$ to $470 \mathrm{~K}$. The suspected parent-body belt location is thus consistent with the prediction that such belts form preferentially before the snowline, $R_{\text {snow }}=2.7\left(L / L_{\odot}\right)^{1 / 2} \mathrm{AU}=11 \mathrm{AU}$, because giant planet accretion is triggered beyond that limit. At such distance, the parent-body population should have eroded in a short timescale compared to the age of the star. Given the properties of the cold dust belt, Poynting-Robertson drag is not a valuable mechanism to transport sufficient amount of material from $\sim 140$ AU down to the warm belt. Even in a very favorable planetary system configuration, scattering of small bodies (comets, asteroids, planetesimals) from the cold belt by a chain of planets could not sustain the required mass production rate in the warm disk for the age of the star.

Another explanation to the unexpectedly large mass in the warm component could be that the dust originates in stochastic and/or isolated catastrophic events, such as planetesimal collisions or break-up, or major dynamical perturbations. In the solar system, the Late Heavy Bombardment (LHB) was responsible for the depletion of the Kuiper belt, the release of large numbers of icy objects into its inner regions and probably a

9 MCFOST uses a 3D geometry: we assume linear flaring, and we test scale heights of $0.001,0.01$ or 0.1 (unitless) at the reference distance of $1 \mathrm{AU}$, with little impact on the results. $r_{\max }$ is fixed to $1 \mathrm{AU}$ and $20 \mathrm{AU}$ for the hot ring and the warm belt respectively. Here the grain temperatures are independent of their sizes. 
durable increase of the inner Zodiacal cloud infrared luminosity (Nesvorný et al. 2010; Booth et al. 2009). The orbital parameters of Fomalhaut $b$ have recently been reevaluated based on an fourth epoch detection with the Hubble Space Telescope (HST). The planet orbit is found to be very eccentric $(\geq 0.8)$ such that it likely approaches the innermost parts of the system at periastron, where additional planetary perturbers might be present (Kalas et al. 2013; Beust et al., in prep.). Thus an LHB-like event may be occurring around Fomalhaut as a result of a planet-planet scattering event causing delayed stirring in both the cold and the warm belt. In summary, a valuable scenario to understand the global debris disk is that a high collisional activity has been triggered by the presence of perturbing planets, reminiscent of the solar system history.

Finally, it is attractive to place our study in the context of the long-term objective of finding and characterizing an Earth-like planet in the habitable zone of a star. Scaling the Mars and Venus criteria for Fomalhaut (Selsis et al. 2007), the habitable zone ranges between 2.5 and $5.5 \mathrm{AU}$. Under favorable $100 \%$ cloudcover conditions, it would extend from 1.4 to 8.1 AU. At these distances, the level of warm dust emission around Fomalhaut is high and represent therefore a threat for future spectroscopic and direct imaging missions (e.g., Defrère et al. 2010; Roberge et al. 2012). In turn, the existence of a massive asteroid belt may be an indication that there is no planet in these region as it would have cleared its neighborhood around its orbit. A noticeable feature that we have not discussed yet is that the gap between the two exozodi components could be sculpted by the gravitational influence of a hidden planet at around 1 AU. Constraints from radial velocities, astrometric measurements, and high-contrast imaging have been summarized in Paper I and are currently compatible with an hypothetical small mass companion in these regions.

\section{Conclusion}

In a series of three papers, we have performed an interferometric study of the Fomalhaut inner debris disk. Paper I presented the detection of a circumstellar excess in $K$-band, attributed to very hot dust, confined well inside the 3 AU-HWHM FOV of the VINCI instrument. Despite the limited spatial constraints, the brightness temperature required calls for extremely hot, refractory grains lying very close to the star, at the sublimation limit. Conversely, KIN null depth measurements presented in Paper II are indicative of a warmer dust component at a few AU (2 AUHWHM FOV) that produces a rising excess upward of $10 \mu \mathrm{m}$. In the present study, we have presented the detailed results of selfconsistent modeling of these two components by means of a parametric radiative transfer code and accurate treatment of debris disk physics. To account for the expected size-dependent sublimation temperature of dust, we introduced a new prescription for the treatment of grain sublimation accounting for their specific dynamics and lifecycle. This enabled us to assess realistically the spatial and size distribution of the emitting grains. We find that the Fomalhaut exozodiacal disk consists of two dust populations, one "classical", though massive, disk of warm ( $\sim 400 \mathrm{~K})$ dust peaking at $\sim 2 \mathrm{AU}$ and declining slowly with distance, responsible for most of the mid-infrared emission, and a hotter $(\sim 2000 \mathrm{~K})$ and brighter counterpart dominated by small $(0.01-0.5 \mu \mathrm{m})$, unbound dust particles at the limit of sublimation. The stellar radius is approximately $9 \times 10^{-3} \mathrm{AU}$ and the hot grains are actually located at typically 10 to 35 stellar radii. The degeneracy inherent to SED fitting is partially broken by the spatial information contained in the interferometric data. We find that the model also fits the photometric mid/far-infrared measurements from Spitzer/MIPS and Herschel/PACS, and is consistent with the flux level measured in the Spitzer/IRS midinfrared spectrum. If the warm dust, or an additional colder (but unresolved) component, were present further out in the system - as suggested by the suspected on-star excess from ALMA - it should produce moderate emission in the mid/farinfrared to preserve the compatibility between the KIN and Herschel/Spitzer data.

We analytically explored the various processes that can affect a dust grain: photo-gravitational and Poynting-Robertson drag forces, collisions, sublimation and disruption of big aggregates. We propose a framework for interpreting self-consistently the simultaneous prevalence of both hot and warm dust in the inner regions of Fomalhaut, similar to that also reported for samples of nearby main sequence stars by near- and mid- infrared exozodi surveys. First, we find that neither of the two inner belts can be explained by a steady-state collisional cascade in a parent-body reservoir. Ignoring the production mechanism for the warm dust, we estimate that PR drag, from this component down to the sublimation radius, could transport enough mass into the hot component, provided that it can accumulate there. We showed that small carbon monomeres released by the disruption of larger aggregates that originate in the warm component can explain the observed flux level in the near-infrared, because this process considerably enhances the effective cross section of the dust population. Finally braking by a gaseous component could preserve these unbound grains from radiative transfer blowout for a sufficient time providing enough gas mass is available. If sublimation is the main source for this gas, it must have accumulated for a timescale comparable to the age of the star.

In summary, the intriguing hot dust phenomenon reported by various interferometric surveys could be understood in the light of the cumulation of multiple effects that eventually yield an accumulation of very small grains at a fraction of an AU. These hot rings are likely the counterparts of warm debris disks orbiting at a few AU that have their dust production triggered by intense collisional activity. In the near future, this scenario will need to be tested against statistical samples of objects, including later type stars.

Acknowledgements. We would like to acknowledge Paul Kalas, James Graham, Kate $\mathrm{Su}$ and Alexis Brandeker, for contributing to interesting discussions on various aspects of this study, as well as the anonymous referee for the valuable advices he provided. The research leading to these results has received funding from the European Community's Seventh Framework Programme under Grant Agreement 226604. We also thank the French National Research Agency (ANR) for financial support through contract ANR-2010 BLAN-0505-01 (EXOZODI), the Programme National de Planétologie (PNP) and the CNES for supporting part of this research.

\section{References}

Absil, O., di Folco, E., Mérand, A., et al. 2006, A\&A, 452, 237

Absil, O., di Folco, E., Mérand, A., et al. 2008, A\&A, 487, 1041

Absil, O., Mennesson, B., Le Bouquin, J.-B., et al. 2009, ApJ, 704, 150

Acke, B., Min, M., Dominik, C., et al. 2012, A\&A, 540, A125

Augereau, J. C., Lagrange, A. M., Mouillet, D., Papaloizou, J. C. B., \& Grorod, P. A. 1999, A\&A, 348, 557

Augereau, J. C., Nelson, R. P., Lagrange, A. M., Papaloizou, J. C. B., \& Mouillet, D. 2001, A\&A, 370, 447

Beichman, C. A., Bryden, G., Gautier, T. N., et al. 2005, ApJ, 626, 1061

Boley, A. C., Payne, M. J., Corder, S., et al. 2012, ApJ, 750, L21

Bonsor, A., \& Wyatt, M. C. 2012, MNRAS, 420, 2990

Bonsor, A., Augereau, J.-C., \& Thébault, P. 2012, A\&A, 548, A104

Booth, M., Wyatt, M. C., Morbidelli, A., Moro-Martín, A., \& Levison, H. F. 2009, MNRAS, 399, 385

Chavero, C., Gómez, M., Whitney, B. A., \& Saffe, C. 2006, A\&A, 452, 921

Coudé du Foresto, V., Borde, P. J., Merand, A., et al. 2003, in SPIE Conf. Ser. 4838 , ed. W. A. Traub, 280 
Currie, T., Debes, J., Rodigas, T. J., et al. 2012, ApJ, 760, L32

Czechowski, A. \& Mann, I. 2010, ApJ, 714, 89

Defrère, D., Absil, O., den Hartog, R., Hanot, C., \& Stark, C. 2010, A\&A, 509, A9

Defrère, D., Absil, O., Augereau, J.-C., et al. 2011a, A\&A, 534, A5

Defrère, D., Absil, O., Augereau, J. C., et al. 2011b, in EPSC-DPS Joint Meeting 2011, 1084

Defrère, D., Lebreton, J., Le Bouquin, J.-B., et al. 2012, A\&A, 546, L9

Dohnanyi, J. S. 1969, J. Geophys. Res., 74, 253

Dominik, C., \& Decin, G. 2003, ApJ, 598, 626

Draine, B. T. 2003, ARA\&A, 41, 241

Fernández, R., Brandeker, A., \& Wu, Y. 2006, ApJ, 643, 509

Galicher, R., Marois, C., Zuckerman, B., \& Macintosh, B. 2013, ApJ, 769, 42

Grün, E. 2007, Encyclopedia of the Solar System, second edition, eds. L.-A.

McFadden, P. R. Weissman, \& T. V. Johnson (San Diego: Academic Press), 621

Hauschildt, P. H., Allard, F., \& Baron, E. 1999, ApJ, 512, 377

Janson, M., Carson, J. C., Lafrenière, D., et al. 2012, ApJ, 747, 116

Kalas, P., Graham, J. R., \& Clampin, M. 2005, Nature, 435, 1067

Kalas, P., Graham, J. R., Chiang, E., et al. 2008, Science, 322, 1345

Kalas, P., Graham, J. R., Fitzgerald, M. P., \& Clampin, M. 2013, ApJ, submitted [arXiv: 1305.2222]

Kama, M., Min, M., \& Dominik, C. 2009, A\&A, 506, 1199

Kobayashi, H., \& Tanaka, H. 2010, Icarus, 206, 735

Kobayashi, H., Watanabe, S.-I., Kimura, H., \& Yamamoto, T. 2008, Icarus, 195, 871

Kobayashi, H., Watanabe, S.-I., Kimura, H., \& Yamamoto, T. 2009, Icarus, 201, 395

Kobayashi, H., Kimura, H., Watanabe, S.-I., Yamamoto, T., \& Müller, S. 2011, Earth Planets Space, 63, 1067

Kobayashi, H., Kimura, H., \& Yamamoto, S. 2013, A\&A, 550, A72

Krivov, A. V., Löhne, T., \& Sremčević, M. 2006, A\&A, 455, 509
Krüger, H., Dikarev, V., Anweiler, B., et al. 2010, Planet. Space Sci., 58, 951

Lamy, P. L. 1974, A\&A, 35, 197

Lawler, S. M., Beichman, C. A., Bryden, G., et al. 2009, ApJ, 705, 89

Le Bouquin, J.-B., Berger, J.-P., Lazareff, B., et al. 2011, A\&A, 535, A67

Lebreton, J., Augereau, J.-C., Thi, W.-F., et al. 2012, A\&A, 539, A17

Lisse, C. M., Wyatt, M. C., Chen, C. H., et al. 2012, ApJ, 747, 93

Löhne, T., Augereau, J.-C., Ertel, S., et al. 2012, A\&A, 537, A110

Mamajek, E. E. 2012, ApJ, 754, L20

Mennesson, B., Absil, O., Lebreton, J., et al. 2013, ApJ, 763, 119

Meyer-Vernet, N., Maksimovic, M., Czechowski, A., et al. 2009, Sol. Phys., 256, 463

Millan-Gabet, R., Serabyn, E., Mennesson, B., et al. 2011, ApJ, 734, 67

Nesvorný, D., Jenniskens, P., Levison, H. F., et al. 2010, ApJ, 713, 816

Pinte, C., Ménard, F., Duchêne, G., \& Bastien, P. 2006, A\&A, 459, 797

Pinte, C., Harries, T. J., Min, M., et al. 2009, A\&A, 498, 967

Quillen, A. C. 2006, MNRAS, 372, L14

Rappaport, S., Levine, A., Chiang, E., et al. 2012, ApJ, 752, 1

Roberge, A., Chen, C. H., Millan-Gabet, R., et al. 2012, PASP, 124, 799

Rowan-Robinson, M., \& May, B. 2013, MNRAS, 533

Selsis, F., Kasting, J. F., Levrard, B., et al. 2007, A\&A, 476, 1373

Stapelfeldt, K. R., Holmes, E. K., Chen, C., et al. 2004, ApJS, 154, 458

Stark, C. C., Kuchner, M. J., Traub, W. A., et al. 2009, ApJ, 703, 1188

Su, K. Y. L., Rieke, G. H., Malhotra, R., et al. 2013, ApJ, 763, 118

Thébault, P., \& Augereau, J. 2007, A\&A, 472, 169

Thébault, P., \& Wu, Y. 2008, A\&A, 481, 713

Wyatt, M. C. 2005, A\&A, 433, 1007

Wyatt, M. C., Smith, R., Greaves, J. S., et al. 2007a, ApJ, 658, 569

Wyatt, M. C., Smith, R., Su, K. Y. L., et al. 2007b, ApJ, 663, 365

Zavitsanos, P. D., \& Carlson, G. A. 1973, J. Chem. Phys., 59, 2966

Zubko, V. G., Mennella, V., Colangeli, L., \& Bussoletti, E. 1996, MNRAS, 282, 1321 


\section{Appendix A: The blowout timescale}

Particles with high $\beta$ ratios are removed from the system by radiation pressure. Here, we derive the typical timescale for this process, for the case of $\beta \gg 1$. In this limit, the transverse movement of the particles is small compared to the radial movement, and only the radial acceleration of the particle needs to be considered.

\section{A.1. The gas free case}

We need to consider the forces of gravity and direct radiation pressure (the PR drag force is negligibly small, and we do not consider gas drag at this stage). These forces are given by

$F_{\text {rad }}+F_{\text {grav }}=\frac{(\beta-1) G M_{\star} m}{r^{2}}$.

For small radial displacements $\Delta r$, the acceleration $\ddot{r}=\left(F_{\text {rad }}+\right.$ $\left.F_{\text {grav }}\right) / m$ can be assumed to be independent of $r$, and the displacement as a function of time is given by $\Delta r=\frac{1}{2} \ddot{r} t^{2}$. The resulting timescale is

$t_{\mathrm{dyn}, \Delta \mathrm{r} \rightarrow 0}(\Delta r)=\sqrt{\frac{2 \Delta r r_{\text {release }}^{2}}{G M_{\star}(\beta-1)}}$.

At large distances from the release point, the acceleration tends to zero, and the velocity of the particle approaches a constant: $\dot{r}(r \rightarrow \infty)=\sqrt{2 G M_{\star} \beta / r_{\text {release }}}$ (Lecavelier des Etangs et al. 1998). Hence, for large displacements, the removal happens on a timescale of

$t_{\mathrm{dyn}, \Delta \mathrm{r} \rightarrow \infty}(\Delta r)=\frac{\Delta r}{\dot{r}(r \rightarrow \infty)}=\sqrt{\frac{(\Delta r)^{2} r_{\text {release }}}{2 G M_{\star}(\beta-1)}}$.

Adding Eqs. (A.2) and (A.3) in quadrature leads to the removal timescale

$t_{\text {dyn }}(\Delta r)=\sqrt{\frac{\Delta r r_{\text {release }}}{G M_{\star}(\beta-1)}\left(2 r_{\text {release }}+\frac{\Delta r}{2}\right)}$.
Comparing this equation with a numerical evaluation of the equation of motion shows that its relative errors are less than $5 \%$ for the values of $\beta$ and $r_{\text {release }}$ considered here.

We define $t_{\text {dyn }}$ as the time it takes for a particle to fly from its release point $\left(r_{\text {release }}\right)$ to a point twice that radial distance from the star $\left(2 r_{\text {release }}\right)$. This is motivated by the fact that the small grains are seen in a very narrow radial range, so only the time they spend close to the release point is relevant. Setting $\Delta r=$ $r_{\text {release }}=r$ gives the blowout timescale given by Eq. (31).

\section{A.2. The high gas density case}

For high gas densities, the gas drag force cannot be ignored. Since the particles considered here are small (compared to the mean free path of the gas molecules), and their velocities are low (compared to the sound speed of the gas), the gas drag force is given by the subsonic Epstein drag law. It is given by

$F_{\text {drag }}=-\frac{4 \pi a^{2} \rho_{\mathrm{g}} v_{\mathrm{th}} \Delta v}{3}$,

where $\rho_{\mathrm{g}}$ is the mass density of the gas, $\Delta v$ is the relative speed between the dust grain and the gas, and $v_{\text {th }}$ is the mean thermal speed of the gas. The latter is given by $v_{\text {th }}=\sqrt{8 k_{\mathrm{B}} T_{\mathrm{g}} /\left(\pi \mu_{\mathrm{g}} m_{\mathrm{u}}\right)}$, where $\mu_{\mathrm{g}}$ is the molecular weight of gas molecules, $m_{\mathrm{u}}$ is the atomic mass unit, $k_{\mathrm{B}}$ is the Boltzmann constant, and $T_{\mathrm{g}}$ is the temperature of the gas. To calculate $v_{\mathrm{th}}$, we assume that the gas temperature equals the dust temperature $\left(T_{\mathrm{g}}=T_{\mathrm{d}}\right)$, and that the gas consists of the same molecules as the dust grains $\left(\mu_{\mathrm{g}}=\mu_{\mathrm{d}}\right)$.

For high gas densities, the particles quickly reach the terminal velocity $v_{\text {term }}$, which is found by solving $F_{\text {rad }}+F_{\text {grav }}+F_{\text {drag }}=0$ for $\Delta v$. This gives

$v_{\text {term }}=\frac{G M_{\star}(\beta-1) \rho_{\mathrm{d}} s}{\rho_{\mathrm{g}} v_{\mathrm{th}} r^{2}}$.

In the high gas density case, the average velocity over the radial range $\Delta r$ can be approximated by the terminal velocity. The blowout timescale for high gas densities is then found from $t_{\text {dyn, } \rho_{\mathrm{g}} \rightarrow \infty}\left(\rho_{\mathrm{g}}, \Delta r\right)=\Delta r / v_{\text {term }}$, which leads to Eq. (32). 
Appendix B: Bayesian probability curves
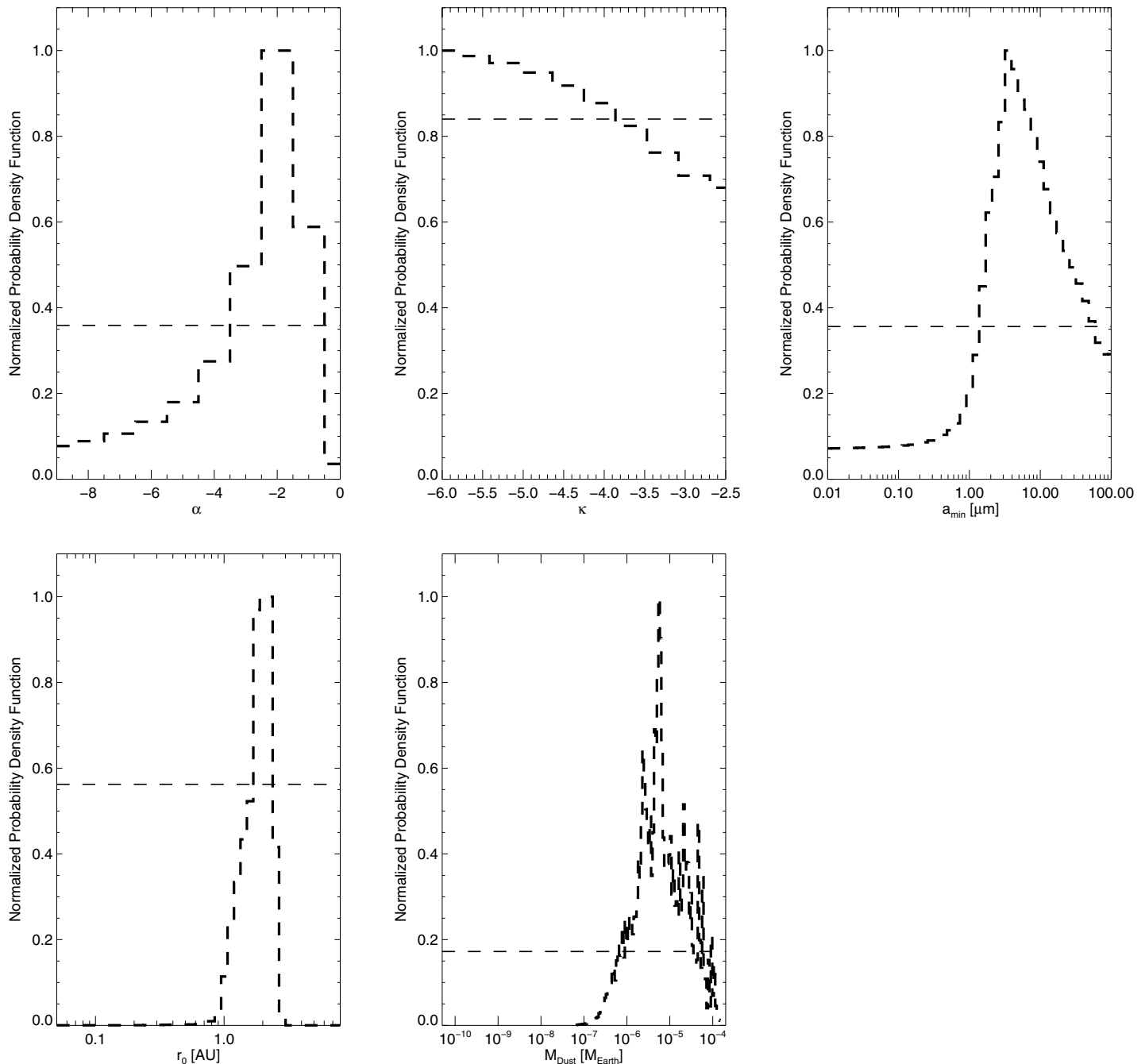

Fig. B.1. Bayesian probability curves obtained when fitting models to the warm component with approach 1 (inner density slope fixed to 10 , free outer density slope $\alpha$ ). 
A\&A 555, A146 (2013)
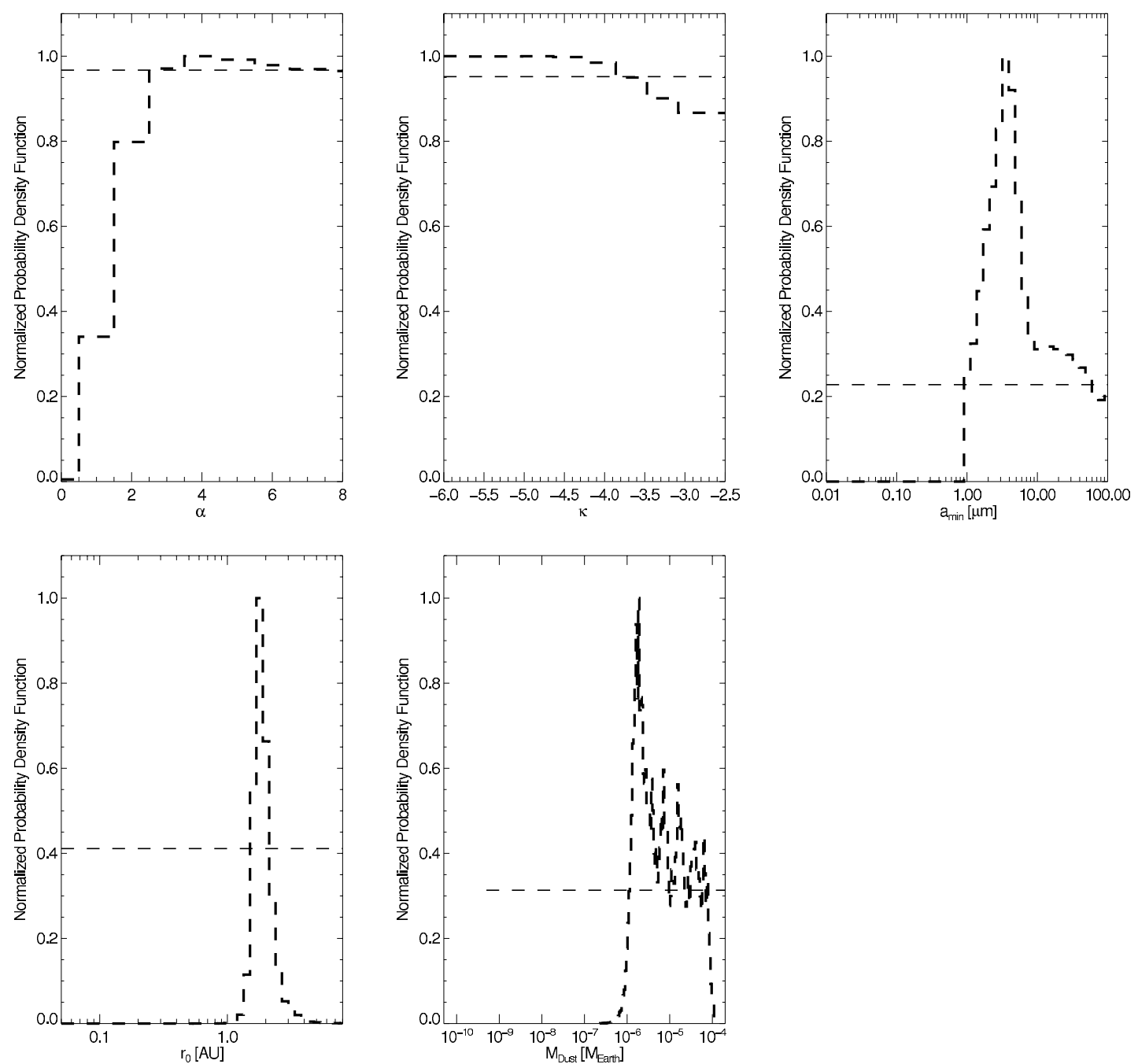

Fig. B.2. Bayesian probability curves obtained when fitting models to the warm component with approach 2 (outer density slope fixed to 1.5 , free inner density slope $\alpha)$. The analysis uses some prior information regarding the grain sizes $P\left(a<a_{\text {blow }} / 10=0\right)$. 